\title{
Interception of host fatty acid metabolism by mycobacteria under hypoxia to suppress anti-TB immunity
}

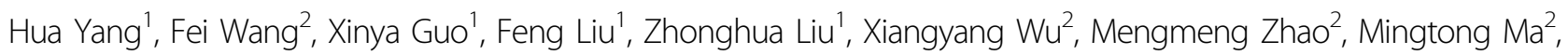

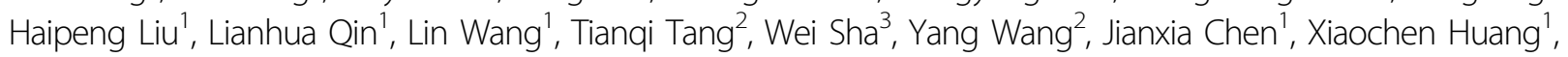
Jie Wang ${ }^{1}$, Cheng Peng ${ }^{2}$, Ruijuan Zheng ${ }^{1}$, Fen Tang ${ }^{2}$, Lu Zhang ${ }^{4}$, Chunyan Wu ${ }^{5}$, Stefan H. Oehlers $\mathbb{D}^{6}$, Zhigang Song ${ }^{7}$, Jialei She ${ }^{7}$, Hua Feng ${ }^{8}$, Xunwei Xie $^{9}$ and Baoxue Ge $\mathbb{1}^{1,2 \otimes}$

\begin{abstract}
Pathogenic mycobacteria induce the formation of hypoxic granulomas during latent tuberculosis (TB) infection, in which the immune system contains, but fails to eliminate the mycobacteria. Fatty acid metabolism-related genes are relatively overrepresented in the mycobacterial genome and mycobacteria favor host-derived fatty acids as nutrient sources. However, whether and how mycobacteria modulate host fatty acid metabolism to drive granuloma progression remains unknown. Here, we report that mycobacteria under hypoxia markedly secrete the protein Rv0859/MMAR_4677 (Fatty-acid degradation A, FadA), which is also enriched in tuberculous granulomas. FadA acts as an acetyltransferase that converts host acetyl-CoA to acetoacetyl-CoA. The reduced acetyl-CoA level suppresses H3K9AC-mediated expression of the host proinflammatory cytokine $1 / 6$, thus promoting granuloma progression. Moreover, supplementation of acetate increases the level of acetyl-CoA and inhibits the formation of granulomas. Our findings suggest an unexpected mechanism of a hypoxia-induced mycobacterial protein suppressing host immunity via modulation of host fatty acid metabolism and raise the possibility of a novel therapeutic strategy for TB infection.
\end{abstract}

\section{Introduction}

Tuberculosis (TB) caused by Mycobacterium tuberculosis (M. tuberculosis) is associated with 10 million active cases and 1.4 million deaths annually ${ }^{1}$. One hallmark of TB is the formation of caseous necrotic granulomas ${ }^{2}$, which are organized aggregates of macrophages and other immune cells that serve as niches for the bacteria to obtain nutrients or evade anti-TB immunity, and provide a source of mycobacteria for later reactivation and dissemination ${ }^{3,4}$.

Low oxygen tension is strongly correlated with the formation of hard, fibrous, and hypoxic granulomas ${ }^{5}$.

Correspondence: Baoxue Ge (gebaoxue@sibs.ac.cn)

${ }^{1}$ Shanghai Key Laboratory of Tuberculosis, Shanghai Pulmonary Hospital,

Tongji University School of Medicine, Shanghai, China

${ }^{2}$ Department of Microbiology and Immunology, Tongji University School of

Medicine, Shanghai, China

Full list of author information is available at the end of the article

These authors contributed equally: Hua Yang, Fei Wang, Xinya Guo, Feng Liu
Mycobacteria must adapt to hypoxia in order to survive within granulomas ${ }^{6-8}$. Hypoxia induces widespread transcriptional changes of mycobacterial genes that are associated with a metabolically altered state and cause the bacteria to enter into a non-replicating "quiescent" state that is tolerant of antibiotic treatment ${ }^{7,9}$. Mycobacteria utilize the ESX-1 type VII and SecA secretion system to transport effector proteins across their cell wall into host immune cells ${ }^{10}$. However, whether and how the secreted mycobacterial proteins are induced under hypoxia to promote the adaption to the hostile environment remains unknown.

The expression profiles of mycobacterial and host genes have been well characterized during granuloma progres$\operatorname{sion}^{11}$. The zebrafish-Mycobacterium marinum (M. marinum) platform has been used to study the role of mycobacterial components including early secretory

\section{(c) The Author(s) 2021}

(c) (i) Open Access This article is licensed under a Creative Commons Attribution 4.0 International License, which permits use, sharing, adaptation, distribution and reproduction cc) in any medium or format, as long as you give appropriate credit to the original author(s) and the source, provide a link to the Creative Commons license, and indicate if changes were made. The images or other third party material in this article are included in the article's Creative Commons license, unless indicated otherwise in a credit line to the material. If material is not included in the article's Creative Commons license and your intended use is not permitted by statutory regulation or exceeds the permitted use, you will need to obtain permission directly from the copyright holder. To view a copy of this license, visit http://creativecommons.org/licenses/by/4.0/. 
antigenic target-6 (ESAT-6), trehalose 6,6'-dimycolate (TDM), and host factors including matrix metalloprotease 9 (MMP9), tumor necrosis factor-alpha (TNFo), and vascular endothelial growth factor (VEGF) in the formation and progression of granulomas ${ }^{12-17}$. However, the signaling mechanisms underlying the interaction of hypoxiainduced mycobacterial secreted proteins with host metabolic factors in the regulation of anti-TB immunity during granuloma progression remain unexplored.

In this work, we profile the hypoxia-induced secreted proteins of $M$. tuberculosis and examine their roles in the progression of granuloma. We show that hypoxia strongly induces the secretion of the mycobacterial Rv0859 (Fattyacid degradation A, FadA) protein, which reduces the level of host acetyl coenzyme A (acetyl-CoA) to suppress histone acetylation-mediated production of proinflammatory cytokines. This in turn promotes the survival of mycobacteria in granuloma for persistent infection.

\section{Results}

\section{Mycobacterial FadA is induced by hypoxia}

To investigate whether hypoxia specifically induces the secretion of mycobacterial proteins, we performed quantitative proteomics analysis (Fig. 1a) of M. tuberculosis strain $\mathrm{H} 37 \mathrm{Rv}$ culture filtrate following the Wayne and Hayes model in vitro ${ }^{18,19}$. Hypoxia increased the production of 22 secreted proteins (Supplementary Table S1) and inhibited the production of 29 secreted proteins (Supplementary Table S2; Fig. 1b). Kyoto Encyclopedia of Genes and Genomes (KEGG) metabolic pathway analysis showed that upregulated proteins under hypoxia were particularly associated with fatty acid metabolism (Supplementary Fig. S1a). Since fatty acid metabolism-related genes are relatively "overrepresented" in the M. tuberculosis genome, and fatty acid metabolism plays an important role in $M$. tuberculosis infection ${ }^{20,21}$, we then focused on the five fatty acid metabolism-related genes Rv0824c, FadA, Rv0860, Rv1094, and Rv3774 for further study. The mRNA levels of these five genes were evaluated, with the expression of FadA being most significantly increased by hypoxia at 7 or 14 days of in vitro culture (Fig. 1c; Supplementary Fig. S1b-e). Consistent with this, the production and secretion of FadA protein in H37Rv or M. marinum Aronson (BAA535) were also significantly induced by hypoxia, as determined by our custom-made anti-FadA-specific polyclonal antibody, positive control anti-ESAT-6 antibody, and negative control anti-SigA antibody (Fig. 1d; Supplementary Fig. S1f). Caseous necrotic granuloma is a hallmark structure of TB that provides a hypoxic environment for the persistent infection of $M$. tuberculosis ${ }^{4,7,9,22-24}$. Infection of zebrafish with $M$. marinum provides a wellestablished model of hypoxic and necrotic tuberculous granuloma $^{23}$. Immunohistochemical staining of granulomas from TB patients and M. marinum-infected adult zebrafish revealed that FadA was highly enriched in the multinucleated giant cells around the center of solid granulomas or caseous granulomas, but not detected in the pathological sections of granulomas from lung cancer patients or whole fish sections of uninfected adult zebrafish, in contrast to the findings for ESAT-6, SigA and isotype controls (Fig. 1e; Supplementary Fig. S1g, h). Together, these results demonstrate that FadA is a hypoxia-induced $M$. tuberculosis protein.

\section{FadA enhances granuloma necrosis}

Considering the high abundance of FadA in granulomas and its specific induction under hypoxia, we next examined the functional relevance of FadA during the formation of tuberculous granulomas. We deleted FadA from M. marinum to generate a mutant $M$. marinum ( $\triangle$ FadA), and complemented M. marinum ( $\triangle \mathrm{FadA})$ with FadA to generate a FadA complemented strain $(\triangle \mathrm{FadA}+\mathrm{FadA})$ (Fig. 2a), whose growth rate under either aeration or hypoxia showed no difference from that of wild-type (WT) M. marinum (Fig. 2b). In accordance with previously described methods ${ }^{15}$, acid fast staining of granulomas was carried out to estimate the bacterial burden in granulomas, and hematoxylin and eosin (H\&E) staining was used to score granulomas for the presence of necrotic regions. As reported previously ${ }^{2,3}$, necrotic granulomas show a greater tendency to progress than solid ones. Granulomas of different M. marinum-infected adult zebrafish scored for M. marinum burden as less than 10 or 10 or more bacteria and the percentage of necrotic granulomas were quantified and compared based on the staining results. The total number of granulomas for each strain was counted and shown with " $n$ ", and the different number of granulomas between different groups also reflected the pathology of $M$. marinum-infected zebrafish. Adult zebrafish infected with $M$. marinum ( $\Delta$ FadA) had a much lower bacterial burden at 14 days post infection and an increased fraction of low-burden or non-necrotic granulomas than WT $M$. marinum or $M$. marinum $(\triangle \mathrm{FadA}+$ FadA $)$ strains (Fig. 2c-f).

It has also been shown that $\mathrm{C} 3 \mathrm{HeB} / \mathrm{FeJ}$ mice infected with $M$. tuberculosis develop well-circumscribed TB lung granulomas with central necrosis and tissue hypoxia ${ }^{25}$. We generated a FadA deletion mutant of $M$. tuberculosis strain H37Rv $\Delta$ FadA and a FadA complemented strain H37Rv $(\Delta \mathrm{FadA}+\mathrm{FadA})$ (Supplementary Fig. S2a), whose growth rate also showed no difference from that of H37Rv (Supplementary Fig. S2b). The lung tissues from $\mathrm{C} 3 \mathrm{HeB} / \mathrm{Fe} J$ mice 4 weeks post infection with the $H 37 R v \Delta$ FadA strain had a much lower bacterial burden (the difference in $\log _{10}$ means between $\mathrm{H} 37 \mathrm{Rv}$ and $\mathrm{H} 37 \mathrm{Rv} \Delta \mathrm{FadA}$ was $-1.575 \pm 0.04916$; the difference in $\log _{10}$ means between $\mathrm{H} 37 \mathrm{Rv} \Delta \mathrm{FadA}$ and $\mathrm{H} 37 \mathrm{Rv}(\Delta \mathrm{FadA}+\mathrm{FadA})$ was $-1.147 \pm$ 0.04838) and far fewer pathological lesions than those 
a

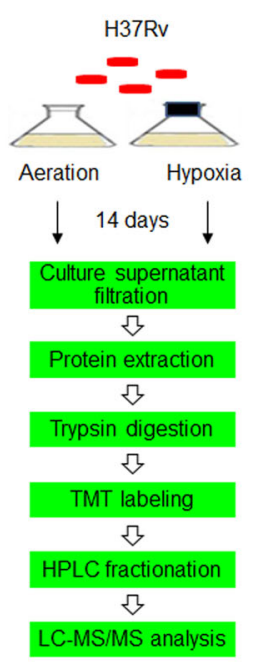

b

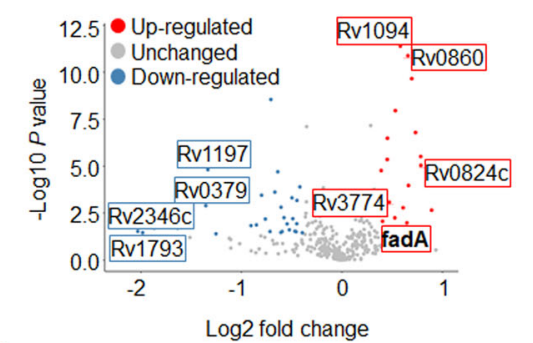

C

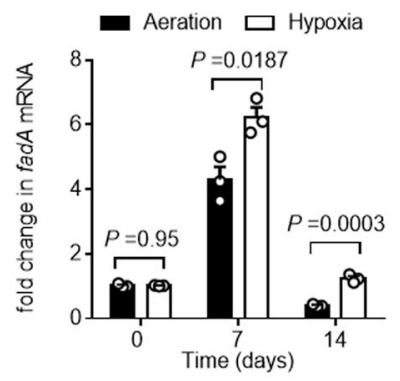

M. tuberculosis

d

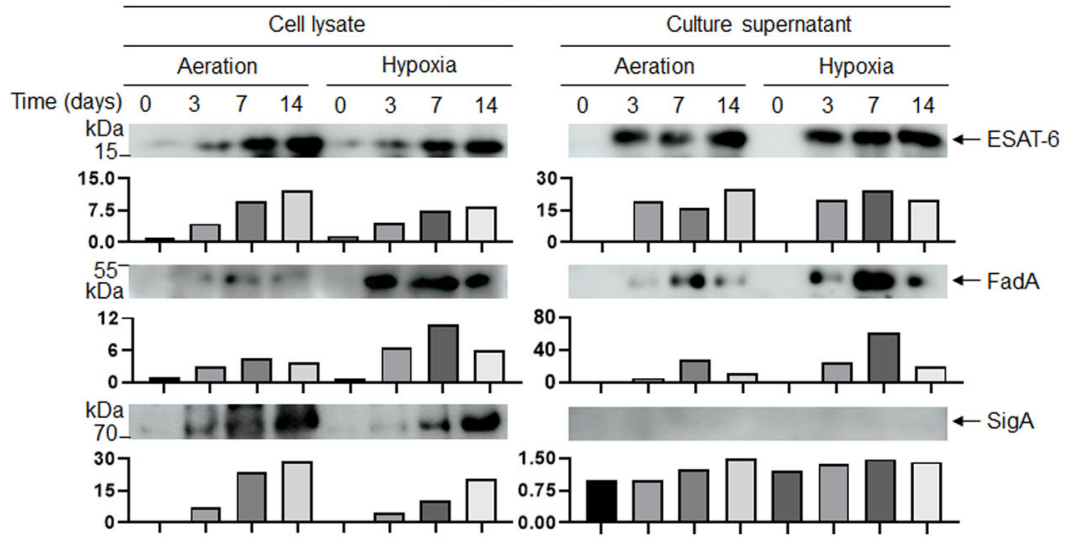

e

TB patient
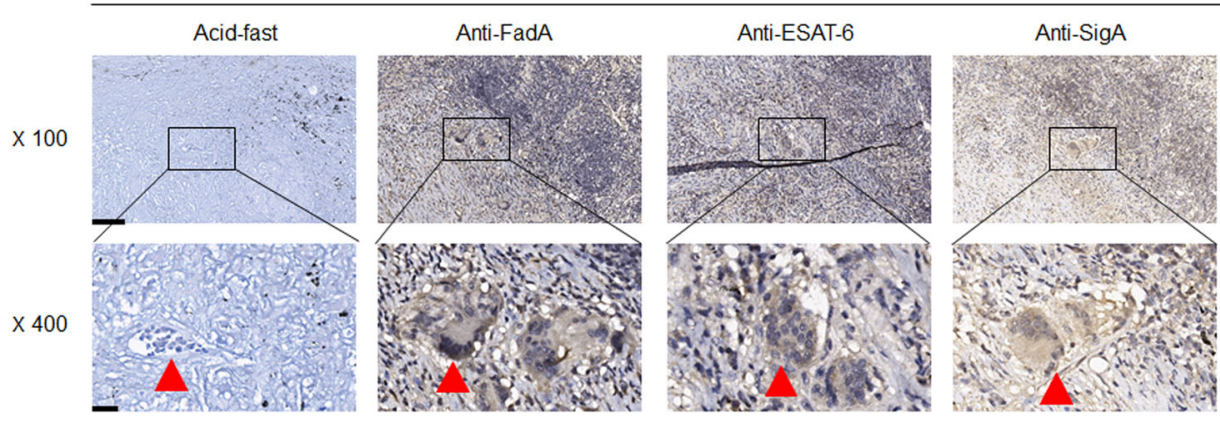

Isotype control

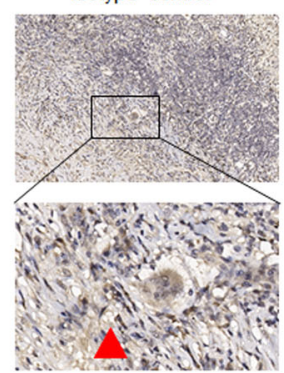

Adult zebrafish
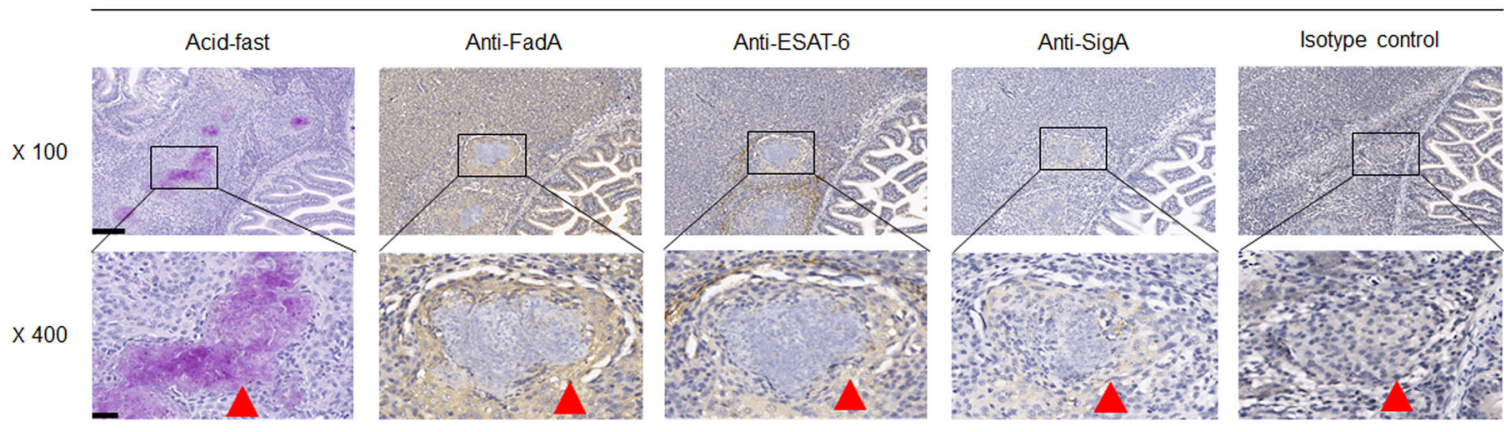

Fig. 1 (See legend on next page.)

infected with the WT $\mathrm{H} 37 \mathrm{Rv}$ or $\mathrm{H} 37 \mathrm{Rv}(\Delta \mathrm{FadA}+\operatorname{FadA})$ strain (Supplementary Fig. S3). Together, these results demonstrate that FadA enhances the survival of mycobacteria in infected zebrafish or mice.

\section{FadA inhibits IL-6}

To investigate the mechanism by which FadA enhances the survival of mycobacteria and granuloma progression, we examined whether FadA had any effect on the 
(see figure on previous page)

Fig. 1 Hypoxia induces FadA. a The pathway of quantitative proteomic analysis to examine alterations in the protein profile of H37Rv culture filtrate using the Wayne model of aeration or hypoxia in vitro. $\mathbf{b}$ Volcano map of identified secreted proteins based on the fold change (Log2) and $t$ test $P$ value (-Log10) by comparing hypoxia with aeration. c qPCR analysis of Rv0859 (fadA) mRNA from H37Rv incubated under aeration and hypoxia for 0, 7, or 14 days in vitro (mean \pm SEM). Data are representative of one experiment with at least three independent biological replicates; each circle represents one technical repeat. Bar charts show means. d Immunoblot (IB) of culture filtrate and cell lysate from H37Rv incubated under aeration and hypoxia for , 3, 7, or 14 days with anti-FadA, anti-ESAT-6, or anti-SigA antibodies at a 1:1000 dilution. Bars represent densitometric analysis of band intensity. e Immunolocalization of FadA in lung granuloma sections from TB patients and whole fish sections of M. marinum infected adult zebrafish with anti-FadA polyclonal antibody at a 1:100 dilution and anti-rabbit secondary antibody labeled with HRP at a 1:200 dilution (scale bar, $100 \mu \mathrm{m}$ (top) and $20 \mu \mathrm{m}$ (bottom)), compared with anti-ESAT-6 polyclonal antibody at a 1:200 dilution, isotype polyclonal control antibody at a 1:100 dilution, anti-SigA antibody labeled with HRP at 1:100 dilution and acid-fast staining (scale bar, $100 \mu \mathrm{m}$ (top) and $20 \mu \mathrm{m}$ (bottom)). The red triangle indicates multinucleated giant cells. Results of $\mathbf{d}$, e are representative of three independent experiments. Two-tailed unpaired Student's $t$ test (c) was used for statistical analysis.

expression of proinflammatory cytokines. Peritoneal macrophages were infected with $\mathrm{H} 37 \mathrm{Rv}, \mathrm{H} 37 \mathrm{Rv} \Delta \mathrm{FadA}$, or $\mathrm{H} 37 \operatorname{Rv}(\Delta \mathrm{FadA}+\mathrm{FadA})$ strain, and the expression of $I l 1 b$, $I l 6, I l 12 b$, and $T n f \alpha$ was analyzed by quantitative real-time polymerase chain reaction (qRT-PCR). Deletion of FadA in $\mathrm{H} 37 \mathrm{Rv}$ markedly increased the expression of $I l 6$, but not that of other cytokines in macrophages, which was also upregulated in the adult zebrafish infected with $\Delta$ FadA $M$. marinum compared with the levels in WT and FadAcomplemented strains (Fig. 3a, b; Supplementary Fig. S4).

IL-6 was previously reported to be essential for anti-TB immunity $^{26}$. Given that FadA enhances the survival of mycobacteria in tuberculous granulomas, and that FadA suppresses the expression of $I l 6$, we next examined the functional relevance of IL-6 for the survival of mycobacteria in vivo. We generated an il6 deletion mutant zebrafish allele using CRISPR/Cas9 genome editing mutagenesis $^{27}$ (Supplementary Fig. S5; Fig. 3c). Deletion of il6 markedly increased $M$. marinum burden compared with that in WT zebrafish (Fig. 3d), which is consistent with the protective immunity conferred by IL- 6 against $M$. tuberculosis infection in mice ${ }^{26}$. Interestingly, deletion of the il 6 gene increased the proportion of necrotic granulomas compared with that in WT zebrafish (Fig. 3e-g), suggesting a previously unidentified role of IL-6 in the inhibition of tuberculous granuloma necrosis. Moreover, the reductions in the burden and rate of granuloma necrosis in WT fish infected with the $M$. marinum ( $\triangle \mathrm{FadA}$ ) mutant compared with the levels in the WT $M$. marinum or $M$. marinum $(\triangle \mathrm{FadA}+\mathrm{FadA})$ strain were not found in il6-deficient mutant zebrafish (Fig. 3d-g).

In parallel with this, we evaluated the effects of IL-6 on granuloma formation in $\mathrm{C} 3 \mathrm{HeB} / \mathrm{FeJ}$ mice infected with $\mathrm{H} 37 \mathrm{Rv}$. The $\mathrm{C} 3 \mathrm{HeB} / \mathrm{FeJ}$ mice were infected with $\mathrm{H} 37 \mathrm{Rv}$, $\mathrm{H} 37 \mathrm{Rv} \Delta \mathrm{FadA}$, or $\mathrm{H} 37 \mathrm{Rv}(\Delta \mathrm{FadA}+\mathrm{FadA})$ strain, and then treated with a neutralizing anti-IL-6 or isotype-matched control monoclonal Ab (mAb) (Supplementary Fig. S6a). Neutralization of IL-6 markedly increased the bacterial burden and histological score of lesions in the lungs of $\mathrm{C} 3 \mathrm{HeB} / \mathrm{FeJ}$ mice infected with $\mathrm{H} 37 \mathrm{Rv}$ (Supplementary Fig. S6b-d). Furthermore, treatment with anti-IL-6 mAb eliminated the reductions in bacterial burden and pathological score seen in $H 37 \mathrm{Rv} \Delta$ FadA-infected mice compared with those in $\mathrm{H} 37 \mathrm{Rv}$ or $\mathrm{H} 37 \mathrm{Rv}(\Delta \mathrm{FadA}+\mathrm{FadA})$ control strain (Supplementary Fig. S6b-d). Together, these results demonstrate that increased host IL-6 mediates the enhanced killing of $H 37 \operatorname{Rv} \Delta$ FadA.

\section{FadA suppresses IL-6 through H3K9AC}

We next investigated the mechanism underlying the suppression of host IL-6 by mycobacterial FadA. There is accumulating evidence that the acetylation of histone proteins plays an important role in reversible gene expression changes $^{28-31}$. From the BIOGPS database, we found that the expression of histone deacetylases (HDAC) 1-3 was higher than that of other HDACs in macrophages (BioGPS). To investigate whether FadA regulates Il6 expression through modulation of host histone acetylation, peritoneal macrophages were pretreated with CI994, an inhibitor of HDAC1- $3^{32}$, and infected with H37Rv, $\Delta$ FadA, or $H 37 \operatorname{Rv}(\Delta$ FadA + FadA $)$ strain. Treatment with CI-994 markedly induced the transcription of Il6 in macrophages infected with H37Rv, but not the control genes (Fig. 4a; Supplementary Fig. S7a, b), suggesting a previously unidentified role of CI-994-sensitive histone acetylation in the regulation of $M$. tuberculosis-induced proinflammatory responses. The inhibitory effect of FadA on the expression of $I l 6$ in macrophages infected with $\mathrm{H} 37 \mathrm{Rv}$ or $\mathrm{H} 37 \mathrm{Rv}(\Delta \mathrm{FadA}+\mathrm{FadA})$ strain was abrogated by the treatment with CI-994 (Fig. 4a). Then, we pretreated macrophages with OSS_128167, a selective inhibitor that increases the acetylation of $\mathrm{H} 3 \mathrm{~K}^{33}$, and infected them with the same strains. The data further confirmed that FadA suppresses $I l 6$ directly by modifying the acetylation of H3K9 (Fig. 4b).

Lysine acetylation on the tails of histone $\mathrm{H} 3$ regulates cytokine-specific transcription during the innate immune response $^{34}$. Our chromatin immunoprecipitationsequencing (ChIP-seq) analysis of histone $\mathrm{H} 3$ acetylation at lysine 9 (H3K9Ac), a histone marker associated with active transcription, showed that the deletion of FadA led to increased enrichment of H3K9Ac surrounding host 
a

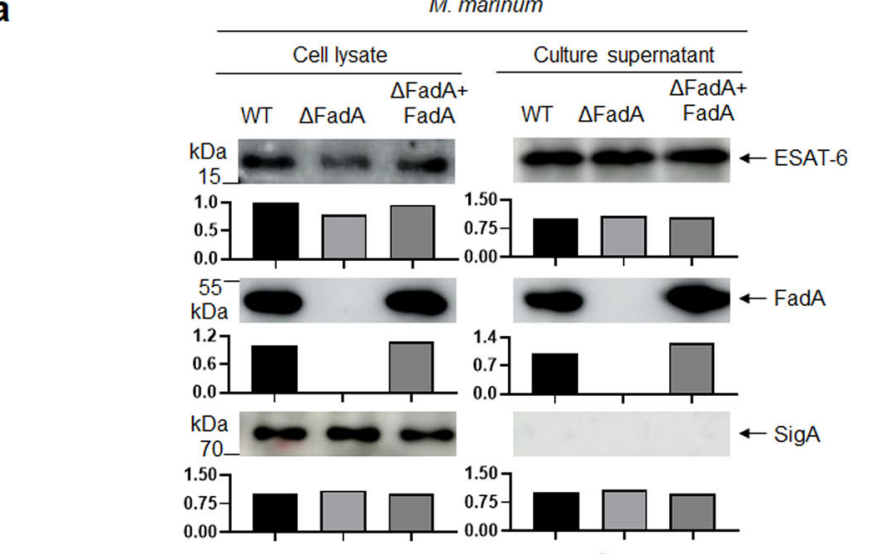

C

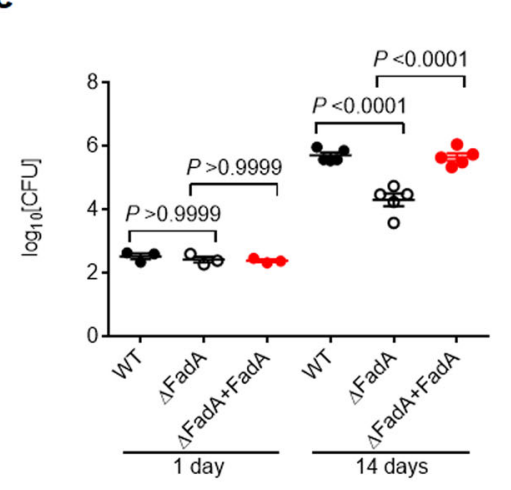

d

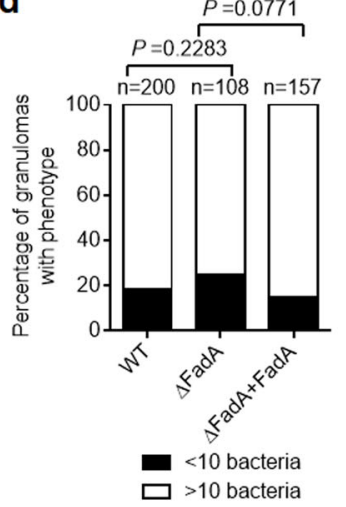

b

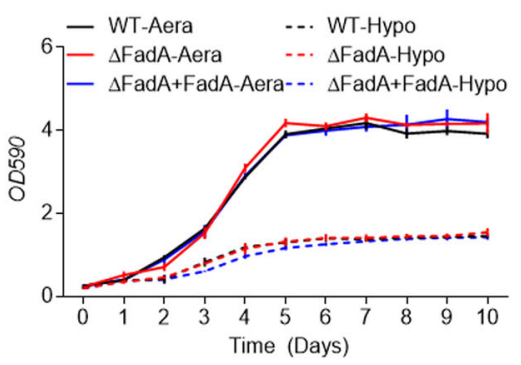

e

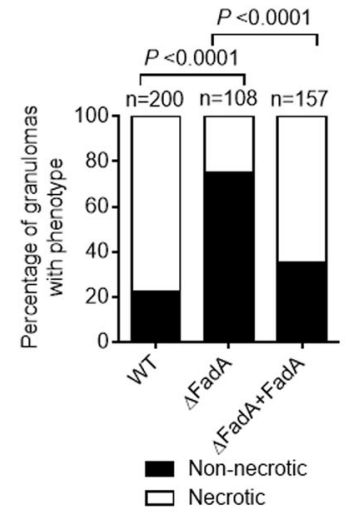

f

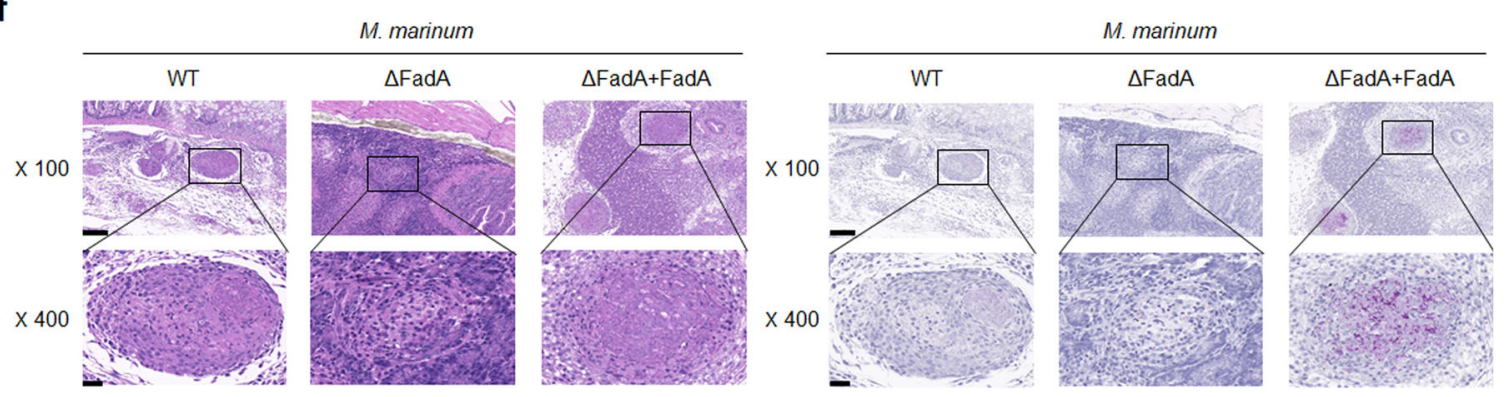

Fig. 2 FadA enhances the survival of mycobacteria in granulomas. a IB of cell lysate and culture filtrate of WT, $\triangle$ FadA, or $\triangle F a d A+F a d A M$. marinum strains with anti-FadA, anti-ESAT-6, and anti-SigA antibodies at a 1:1000 dilution. Bars represent densitometric analysis of band intensity. Results are representative of three independent experiments. $\mathbf{b}$ Growth curve for in vitro growth of WT, $\triangle$ FadA, or $\triangle$ FadA + FadA M. marinum strains under aeration or hypoxia at $30^{\circ} \mathrm{C}$ for 10 days. c-f Adult zebrafish were intraperitoneally infected with roughly $200 \mathrm{CFU}$ per fish of WT, $\triangle$ FadA, or $\triangle$ FadA + FadA M. marinum strains for 1 or 14 days. Histopathology was assessed in the whole fish sections with bacterial titers ( $\mathbf{c}$; mean \pm SEM of $n=$ 3 fish infected for 1 day or $n=5$ fish infected for 14 days), comparison of granulomas between WT, $\Delta$ FadA, or $\triangle$ FadA + FadA M. marinum-infected adult zebrafish scored for M. marinum burden as less than 10 or 10 or more bacteria (d), or percentage of necrotic granulomas in each fish (e) and H\&E or acid-fast staining from zebrafish infected for 14 days (f; scale bar, $100 \mu \mathrm{m}$ (top) and $20 \mu \mathrm{m}$ (bottom)). " $n$ " in d, e was the total number of granulomas for each strain infected fish. A total number of zebrafish analyzed: five $(W T)$, five $(\Delta$ FadA), five $(\Delta$ FadA + FadA). Data in $\mathbf{b}-\mathbf{f}$ represent one experiment with at least three independent replicates. One-way ANOVA with Bonferroni's multiple comparisons test (c) and Fisher's exact test (d, e) was used for statistical analysis.

genome transcription start sites (TSSs) in macrophages infected with H37Rv (Supplementary Fig. S8). Compared with the case for the constitutively active Rpl30 locus, deletion of FadA markedly increased the amount of H3K9Ac modification in the $I l 6$ promoter in H37Rvinfected macrophages (Fig. 4c, d), suggesting that FadA may inhibit M. tuberculosis-induced expression of $I l 6$ through reducing the level of H3K9Ac.

\section{FadA reduces acetyl-CoA}

Histone acetylation utilizes acetyl coenzyme A (acetyl$\mathrm{CoA})$ as a substrate ${ }^{35,36}$. According to the three-dimensional 


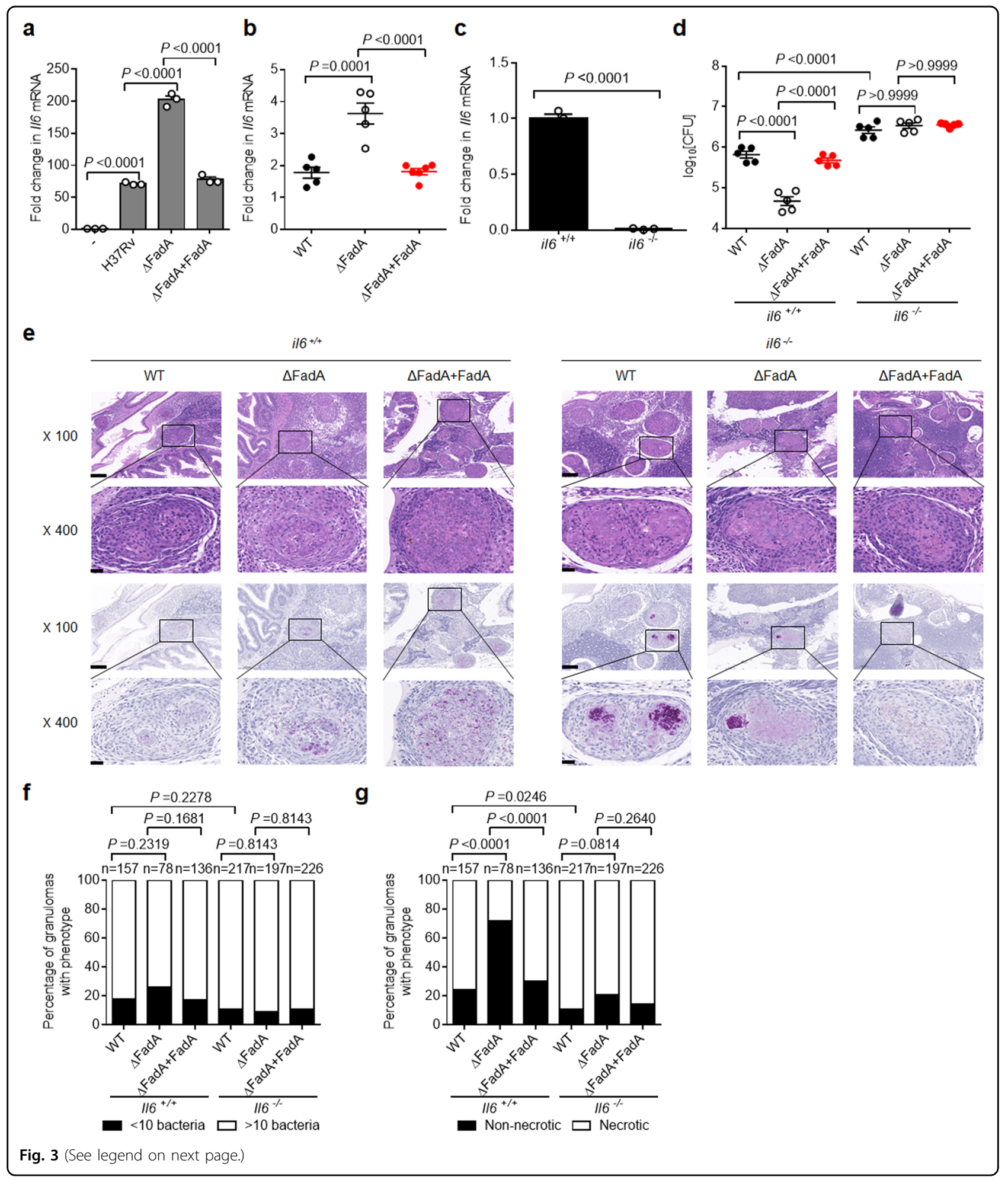

structure of FadA (PDB code: 4B3H $)^{37}$, FadA appears to be an acyl-CoA thiolase, a key enzyme for the fourth step of reaction in the fatty acid $\beta$-oxidation pathway involving M. tuberculosis, which can catalyze the reversible thiolytic cleavage of 3-ketoacyl-CoA to acyl-CoA and acetyl$\mathrm{CoA}^{38}$. However, the conserved domain alignment analysis revealed FadA to be an acetyl-CoA acetyltransferase that can catalyze the synthesis of acetoacetyl-CoA 
(see figure on previous page)

Fig. 3 FadA inhibits host immune responses. a qPCR analysis of 116 mRNA from peritoneal macrophages infected with H37Rv, H37Rv $\triangle F a d A$, or $\mathrm{H} 37 \mathrm{Rv}(\triangle \mathrm{FadA}+\mathrm{FadA})$ strains for $4 \mathrm{~h}(\mathrm{MOI}=1)($ mean $\pm \mathrm{SEM})$. b qPCR analysis of $116 \mathrm{mRNA}$ from adult zebrafish intraperitoneally infected with roughly 200 CFU per fish of WT, $\triangle F$ FadA, or $\triangle$ FadA + FadA M. marinum strains for 14 days (mean \pm SEM of $n=5$ ). c qPCR analysis of 116 mRNA from $i / 6^{+/+}$and $i / 6^{-1-}$ zebrafish (mean \pm SEM). The Cas9/gRNA system was employed to generate IL- 6 knockout zebrafish, which were constructed at the China Zebrafish Resource Center (CZRC) as described previously. $\mathbf{d}-\mathbf{g} i / 6^{+/+}$and $i / 6^{-/-}$mutant adult zebrafish were intraperitoneally infected with approximately $200 \mathrm{CFU}$ of WT, $\triangle \mathrm{FadA}$, or $\triangle \mathrm{FadA}+$ FadA M. marinum strains for 14 days. Bacterial titers (d; mean \pm SEM of $n=5$ fish infected for 14 days), histopathology (e; representative of one experiment with at least three independent replicates; scale bar, $100 \mu \mathrm{m}$ (top) and $20 \mu \mathrm{m}$ (bottom)), and comparison of granulomas between WT, $\triangle$ FadA, or $\triangle$ FadA + FadA M. marinum-infected $i / 6^{+/+}$and $i / 6^{-/-}$adult zebrafish scored for M. marinum burden as less than 10 or 10 or more bacteria $(\mathbf{f})$ or percentage of necrotic granulomas in each fish $(\mathbf{g})$ were assessed as described previously. " $n$ " was the total number of granulomas for each strain infected fish. Total number of zebrafish analyzed: five $\left(\mathrm{WT} / \mathrm{il}^{+/+}\right)$, five $\left(\Delta \mathrm{FadA} / \mathrm{il} 6^{+/+}\right)$, five $(\Delta \mathrm{FadA}$ + FadA/il $\left.6^{+/+}\right)$, five $\left(\mathrm{WT} / \mathrm{il} 6^{-/-}\right)$, five $\left(\Delta \mathrm{FadA} / \mathrm{il} 6^{-/-}\right)$, five $\left(\Delta \mathrm{FadA}+\mathrm{FadA} / \mathrm{il} 6^{-/-}\right)$. Data in $\mathbf{a}-\mathbf{g}$ represent one experiment with at least three independent replicates. One-way ANOVA with Bonferroni's multiple comparisons test (a, b, d), two-tailed unpaired Student's $t$-test (c) and Fisher's exact test $(\mathbf{f}, \mathbf{g})$ were used for statistical analysis.

\section{$\square \mathrm{H} 37 \mathrm{Rv} \square \triangle \mathrm{FadA} \square \triangle \mathrm{FadA}+\mathrm{FadA}$}

a

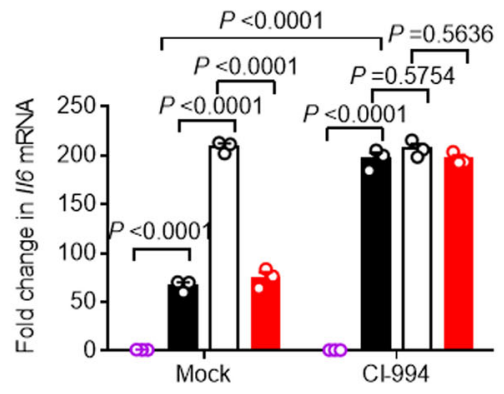

C
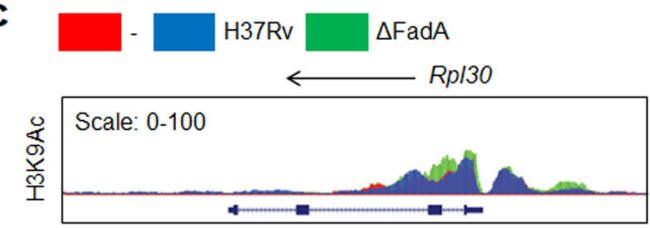

Chr15:34,439,000 $116 \longrightarrow$

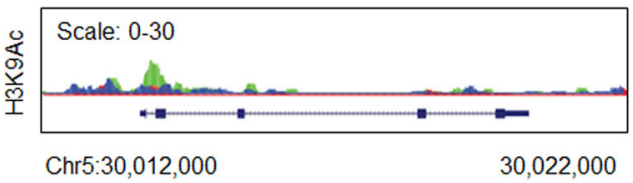

b

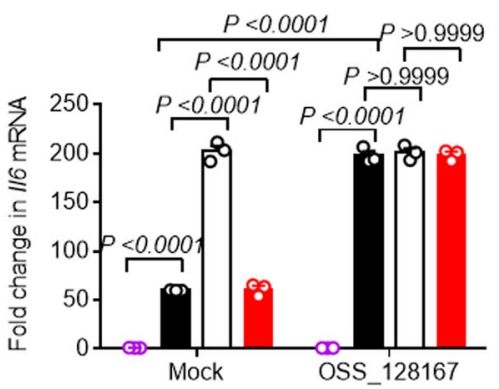

d



Fig. 4 FadA suppresses IL-6 through H3K9Ac. a qPCR analysis of 116 mRNA from control or histone deacetylase (HDAC) 1-3 inhibitor Cl-994pretreated peritoneal macrophages infected with H37Rv, H37Rv $\triangle$ FadA, or H37Rv( $\triangle F a d A+F a d A)$ strains for $4 \mathrm{~h}(\mathrm{MOI}=1)(\mathrm{mean} \pm \mathrm{SEM})$. b qPCR analysis of 116 mRNA from control or OSS_128167, a selective inhibitor that increases the acetylation of H3K9-pretreated peritoneal macrophages infected with H37Rv, H37Rv $\triangle$ FadA, or H37Rv( $\triangle$ FadA + FadA) strains for $4 \mathrm{~h}(\mathrm{MOI}=1)$ (mean \pm SEM). c ChIP-seq analysis of histone H3 acetylation at the lysine 9 residue (H3K9Ac) for the $1 / 6$ promoter of peritoneal macrophage infected with H37Rv or H37Rv $\Delta$ FadA strains for $4 \mathrm{~h}(\mathrm{MOI}=1)$ with SimpleChIP Enzymatic Chromatin IP Kit. d ChIP-qPCR (mean \pm SEM) analysis of H3K9Ac for the $1 / 6$ promoter of peritoneal macrophage infected with negative control, H37Rv, H37Rv $\triangle \mathrm{FadA}$, or H37Rv $(\triangle \mathrm{FadA}+\mathrm{FadA})$ strains for $4 \mathrm{~h}(\mathrm{MOI}=1)$. Data in $\mathbf{a}, \mathbf{b}, \mathbf{d}$ represent one experiment with at least three independent replicates. One-way (d) or two-way $(\mathbf{a}, \mathbf{b})$ ANOVA with Bonferroni's multiple comparisons test were used for statistical analysis.

(AcAcCoA) from two molecules of acetyl-CoA ${ }^{38}$ (Fig. 5a). To examine the acetyl-CoA acetyltransferase activity of FadA, we expressed FadA as a C-terminally His6-tagged protein from Escherichia coli (E. coli) and purified using immobilized metal ion affinity chromatography (Supplementary Fig. S9a). The purified FadA was assayed for synthesis activity with acetyl-CoA as the substrate to yield AcAcCoA and coenzyme A at pH 8.1 ${ }^{39}$. A two-substrate steady-state kinetic assay confirmed that the addition of FadA led to the consumption of acetyl-CoA in solution (Fig. 5b), suggesting that FadA may function as an acetylCoA acetyltransferase. Consistent with this finding, 


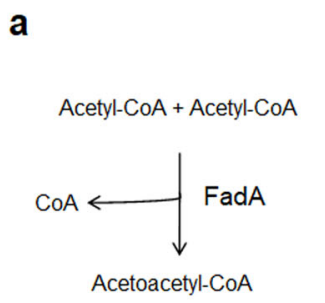

d

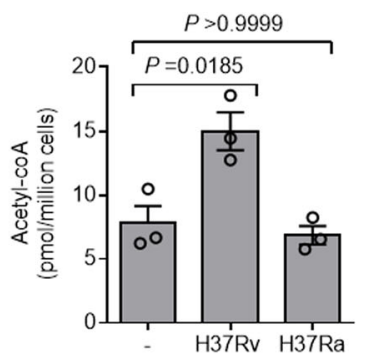

b


C

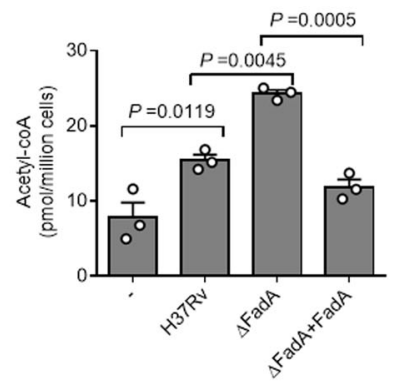

H37Rv $\square \triangle \mathrm{FadA} \square \triangle \mathrm{FadA}+\mathrm{FadA}$ $\stackrel{P<0.0001}{P<0.0001}$

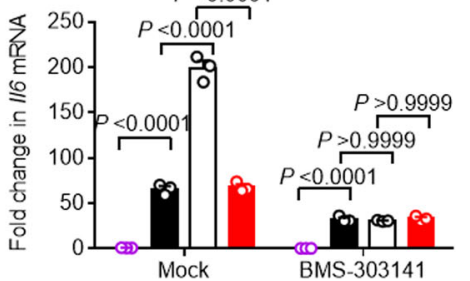

Fig. 5 FadA reduces acetyl-CoA. a Diagram of the acetyl-CoA acetyltransferase activity of FadA. $\mathbf{b}$ In vitro two-substrate steady-state kinetic assay of acetyltransferase activity of recombinant FadA protein. c Acetyl-CoA level assay of peritoneal macrophages infected with H37Rv, H37Rv $\triangle$ FadA or $\mathrm{H} 37 \mathrm{Rv}(\triangle \mathrm{FadA}+\mathrm{FadA})$ strains for $4 \mathrm{~h}(\mathrm{MOI}=1)($ mean $\pm \mathrm{SEM})$. d Acetyl-CoA level assay of peritoneal macrophages infected with $\mathrm{H} 37 \mathrm{Rv}$ or $\mathrm{H} 37 \mathrm{Ra}$ for $4 \mathrm{~h}(\mathrm{MOI}=1)($ mean \pm SEM). e Acetyl-CoA level assay of peritoneal macrophages stimulated with recombinant ESAT-6 protein $(5 \mu \mathrm{g} / \mathrm{mL})$ for $4 \mathrm{~h}$ (mean \pm SEM). $\mathbf{f}$ qPCR analysis of $1 / 6$ mRNA from control or ATP-citrate lyase (ACL) inhibitor BMS-303141-pretreated peritoneal macrophages infected with H37Rv, H37Rv $\triangle F a d A$, or H37Rv $(\triangle \mathrm{FadA}+\mathrm{FadA})$ strains for $4 \mathrm{~h}(\mathrm{MOI}=1)$ (mean $\pm \mathrm{SEM})$. Data in $\mathbf{b}-\mathbf{f}$ are representative of one experiment with at least three independent biological replicates; each circle represents one technical repeat. One-way (c, d) or two-way (f) ANOVA with Bonferroni's multiple comparisons test and two-tailed unpaired Student's t-test (e) were used for statistical analysis.

macrophages infected with $\mathrm{H} 37 \mathrm{Rv} \Delta \mathrm{FadA}$ had a much higher level of acetyl-CoA in their cytoplasm than those infected with $\mathrm{H} 37 \mathrm{Rv}$ or $\mathrm{H} 37 \mathrm{Rv}(\triangle \mathrm{FadA}+\mathrm{FadA})$ strain (Fig. $5 \mathrm{c}$ ). Intriguingly, peritoneal macrophages (Fig. 5d) or PMAdifferentiated THP-1 cells $^{40-42}$ responded to virulent $M$. tuberculosis strain $\mathrm{H} 37 \mathrm{Rv}$ as well as its virulence factor ESAT-6, but not to its avirulent counterpart H37Ra (Fig. 5e), to increase the acetyl-CoA level, which drives the differentiation of foamy macrophages for the provision of nutrients $^{40-42}$. Together, our results suggest that FadA may act as an acetyl-CoA acetyltransferase that restrains host acetylCoA levels in M. tuberculosis-infected macrophages.

To determine whether FadA inhibits the expression of Il6 by reducing cytosolic acetyl-CoA, we treated macrophages with BMS-303141, an inhibitor of ATP-citrate lyase (ACL), which is the enzyme that catalyzes the conversion of citrate to acetyl-CoA ${ }^{43}$. Treatment with BMS303141 markedly reduced the $\mathrm{H} 37 \mathrm{Rv}$-induced expression of $I l 6$, suggesting that acetyl-CoA may enhance the proinflammatory responses to $M$. tuberculosis infection (Fig. 5f). Furthermore, the inhibition of ACL by BMS303141 ablated the inhibitory effect of FadA on the expression of $I l 6$ (Fig. 5f). Taken together, these results suggest that FadA may suppress the anti-TB immunity by reducing cytoplasmic acetyl-CoA.

\section{FadA functions through its acetyltransferase activity}

Analysis of the three-dimensional structure of FadA in M. tuberculosis ${ }^{37}$ identified two key enzymatic sites, H359 and C389, which are evolutionarily conserved in FadA isozymes from mycobacteria and common pathogenic bacteria (Fig. 6a). The H359 and C389 residues of FadA were mutated individually to alanine, and $\operatorname{FadA}(\mathrm{H} 359 \mathrm{~A})$ and $\mathrm{FadA}(\mathrm{C} 389 \mathrm{~A})$ were expressed as recombinant proteins from E. coli (Supplementary Fig. S9a). Mutation of either site abrogated the acetyl transfer activity of FadA (Fig. 6b), suggesting that H359 and C389 are essential for the acetyltransferase activity of FadA. To further investigate whether FadA reduces cytoplasmic acetyl-CoA via H359 or C389, the $\mathrm{H} 37 \mathrm{Rv} \Delta \mathrm{FadA}$ strain was complemented with $\mathrm{FadA}(\mathrm{H} 359 \mathrm{~A})$ or $\mathrm{FadA}(\mathrm{C} 389 \mathrm{~A})$ to generate the H37Rv $(\triangle \mathrm{FadA}+\mathrm{FadA}(\mathrm{H} 359 \mathrm{~A}))$ and $\mathrm{H} 37 \mathrm{Rv}(\Delta \mathrm{FadA}+\mathrm{FadA}$ (C389A)) strains (Supplementary Fig. S9b), and the conserved H373 and C403 residues of FadA in M. marinum were mutated individually to alanine, and the $\triangle \mathrm{FadA}+$ $\operatorname{FadA}(\mathrm{H} 373 \mathrm{~A})$ and $\Delta \mathrm{FadA}+\operatorname{FadA}(\mathrm{C} 403 \mathrm{~A})$ M. marinum strains were constructed based on $M$. marinum ( $\triangle \mathrm{FadA})$ (Supplementary Fig. S9c). The secretion and total synthesis of FadA or various FadA mutants in M. tuberculosis and M. marinum under aeration and hypoxia were examined by western blotting analysis of cell lysates and culture 
a

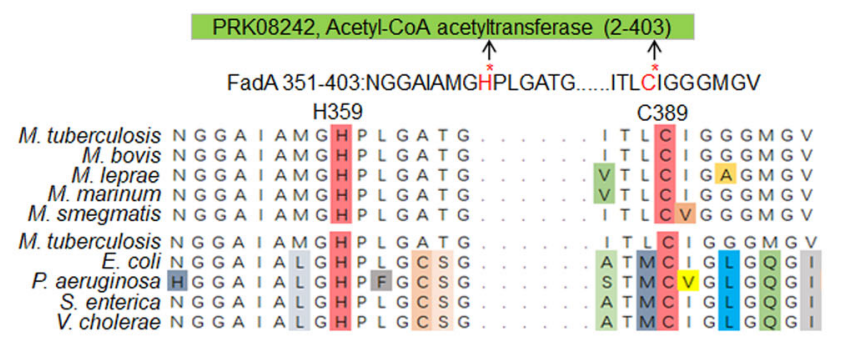

C

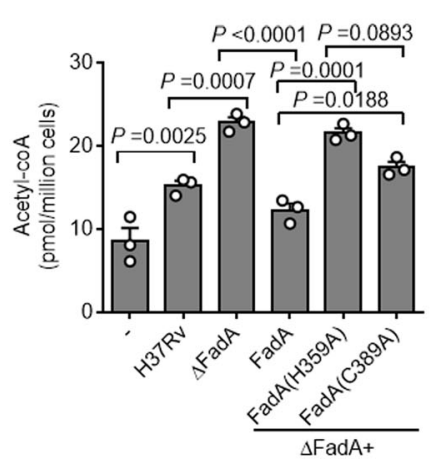

d

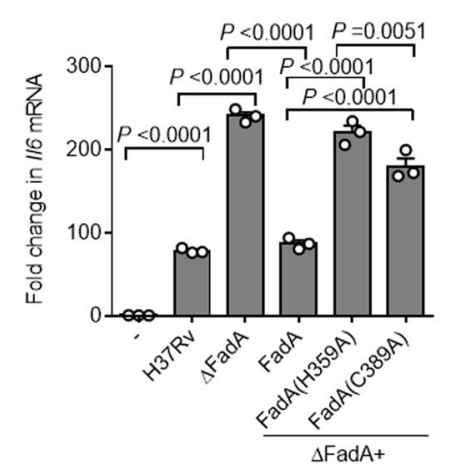

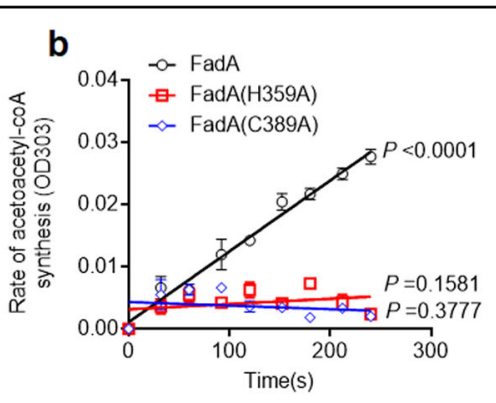

e

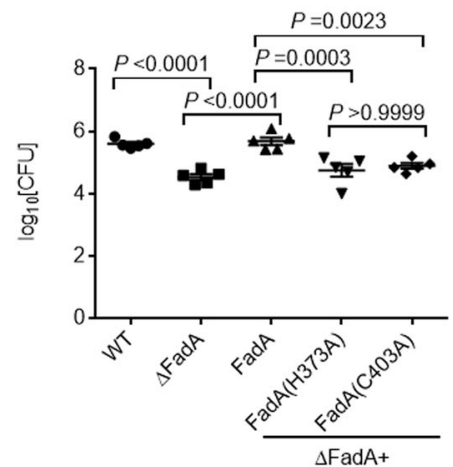

Fig. 6 FadA functions through its acetyltransferase activity. a Alignment of H359 and C389 sites of FadA in mycobacteria and bacteria. b In vitro two-substrate steady-state kinetic assay of acetyltransferase activity of recombinant FadA and FadA-Mut (FadA(H359A), FadA(C389A)) protein. c Assay of the acetyl-CoA level of peritoneal macrophages infected with H37Rv, H37Rv $\triangle F a d A, H 37 R v(\triangle F a d A+F a d A), H 37 R v(\triangle F a d A+F a d A(H 359 A))$, and $\mathrm{H} 37 \mathrm{Rv}(\triangle \mathrm{FadA}+\mathrm{FadA}(\mathrm{C} 389 \mathrm{~A}))$ strains for $4 \mathrm{~h}(\mathrm{MOI}=1)($ mean $\pm \mathrm{SEM}) \cdot \mathbf{d}$ qPCR analysis of $116 \mathrm{mRNA}$ of peritoneal macrophages infected with $\mathrm{H} 37 \mathrm{Rv}$, H37Rv $\triangle F a d A, H 37 R v(\triangle F a d A+F a d A), H 37 R v(\triangle F a d A+F a d A(H 359 A))$, and H37Rv( $(F a d A+F a d A(C 389 A))$ strains for $4 \mathrm{~h}(M O I=1)(m e a n \pm S E M)$. e Adult zebrafish were intraperitoneally infected with roughly 200 CFU of WT, $\triangle$ FadA, $\triangle$ FadA + FadA, $\triangle$ FadA + FadA(H373A), and $\triangle$ FadA + FadA (C403A) M. marinum strains for 14 days. Histopathology was assessed in the whole fish by bacterial titers (mean \pm SEM of $n=5$ fish infected for 14 days). Total number of zebrafish analyzed: five (WT), five ( $\triangle$ FadA), five $(\triangle \mathrm{FadA}+\mathrm{FadA})$, five $(\triangle \mathrm{FadA}+\mathrm{FadA}(\mathrm{H} 373 \mathrm{~A}))$, five $(\triangle \mathrm{FadA}+\mathrm{FadA}(\mathrm{C} 403 \mathrm{~A}))$. Data in b-e represent one experiment with at least three independent replicates. One-way ANOVA with Bonferroni's multiple comparisons test (c-e) was used for statistical analysis.

supernatants. The data showed that the secretion and synthesis of FadA mutants were induced by hypoxia at similar levels to WT FadA (Supplementary Fig. S9d, e). Reconstitution of $H 37 \operatorname{Rv} \Delta$ FadA with FadA, but not the $\operatorname{FadA}(\mathrm{H} 359 \mathrm{~A})$ or $\mathrm{FadA}(\mathrm{C} 389 \mathrm{~A})$ mutants, markedly decreased the level of acetyl-CoA in the M. tuberculosisinfected macrophages (Fig. 6c). These results demonstrate that FadA enzymatically reduces host acetyl-CoA level through H373 and C403 residue-dependent acetyltransferase activity.

To examine whether the enzymatic activity of FadA is also required for its inhibitory effect on the expression of Il6, primary peritoneal macrophages were infected with $\operatorname{H} 37 \operatorname{Rv}(\Delta \operatorname{FadA}+\operatorname{FadA}), \quad \operatorname{H} 37 \operatorname{Rv}(\Delta \mathrm{FadA}+\operatorname{FadA}$ $(\mathrm{H} 359 \mathrm{~A}))$, or $\mathrm{H} 37 \operatorname{Rv}(\triangle \operatorname{FadA}+\operatorname{FadA}(\mathrm{C} 389 \mathrm{~A}))$ strain individually, and the mRNA level of $I l 6$ was examined. The results demonstrated that neither FadA(H359A) nor FadA(C389A) inhibited the expression of $I l 6$ compared with WT FadA (Fig. 6d), indicating that the acetyltransferase activity is essential for FadA to inhibit the host proinflammatory response. Moreover, the
mRNA level of $I l 6$ in all macrophages infected with different $\mathrm{H} 37 \mathrm{Rv}$ strains was increased by the treatment of CI-994 (Supplementary Fig. S10a), but decreased by BMS-303141 (Supplementary Fig. S10b). Together, these results suggest that the inhibitory effect of FadA on the expression of Il6 may require its acetyltransferase activity.

To determine whether FadA promotes the progression of tuberculous granulomas via the two key enzymatic sites, the $\Delta \mathrm{FadA}+\mathrm{FadA}, \Delta \mathrm{FadA}+\operatorname{FadA}(\mathrm{H} 373 \mathrm{~A})$, or $\Delta$ FadA $+\operatorname{FadA}(C 403 \mathrm{~A})$ M. marinum strains were used to infect adult zebrafish. First, the mRNA level of $I l 6$ in the adult zebrafish infected with each $M$. marinum strain for 14 days was examined, and we found that neither FadA(H373A) nor FadA(C403A) inhibited the expression of Il6 compared with WT FadA (Supplementary Fig. S10c). Second, we infected adult zebrafish with the $\Delta$ FadA + FadA,$\quad \Delta$ FadA + FadA $(H 373 \mathrm{~A})$, or $\Delta$ FadA + FadA(C403A) M. marinum strains and demonstrated that reconstitution of $M$. marinum $(\triangle \mathrm{FadA})$ with FadA but not the $\operatorname{FadA}(\mathrm{H} 373 \mathrm{~A})$ or $\operatorname{FadA}(\mathrm{C} 403 \mathrm{~A})$ mutant led 
to a much higher bacterial burden and markedly increased the fraction of necrotic granulomas in adult zebrafish (Fig. 6e; Supplementary Fig. S10d-f). This indicates that FadA promotes the in vivo survival of mycobacteria and necrosis of tuberculous granulomas through its acetyltransferase activity.

\section{Targeting acetyl-CoA for TB therapy}

Host-directed therapy (HDT) has emerged as an attractive strategy for treating $\mathrm{TB}$, especially drugresistant $\mathrm{TB}$, by targeting host pathways to increase anti-TB immunity ${ }^{44}$. Since mycobacterial FadA protein promotes the survival of pathogenic mycobacteria and necrosis of tuberculous granulomas through manipulating host fatty acid metabolism, we next addressed the therapeutic effect of targeting host fatty acid metabolism in adult zebrafish. Acetyl-CoA is known to be produced from acetate by acetyl-CoA synthetase ${ }^{45}$.
Acetate supplementation significantly increased the level of acetyl-CoA in peritoneal macrophages (Fig. 7a), indicating that acetate is an inducer of acetyl-CoA. Consistent with this, treatment with acetate also markedly enhanced the expression of $I l 6$ in H37Rv-infected macrophages (Fig. 7b). Moreover, the addition of acetate abrogated the inhibitory effect of FadA on the expression of Il6 in macrophages infected with H37Rv (Fig. 7b). Similarly, the differential inhibition of WT FadA and its inactive mutants FadA(H359A) or FadA (C389A) on the expression of $I l 6$ in H37Rv-infected macrophages was not observed following the addition of acetate (Fig. 7c). Finally, treatment of acetate markedly reduced the bacterial burden and pathological lesions in the zebrafish infected with $M$. marinum (Fig. $7 \mathrm{~d}-\mathrm{f}$; Supplementary Fig. S11). These results suggest that targeting host fatty acid metabolism may provide an adjunctive therapy for mycobacterial infections.

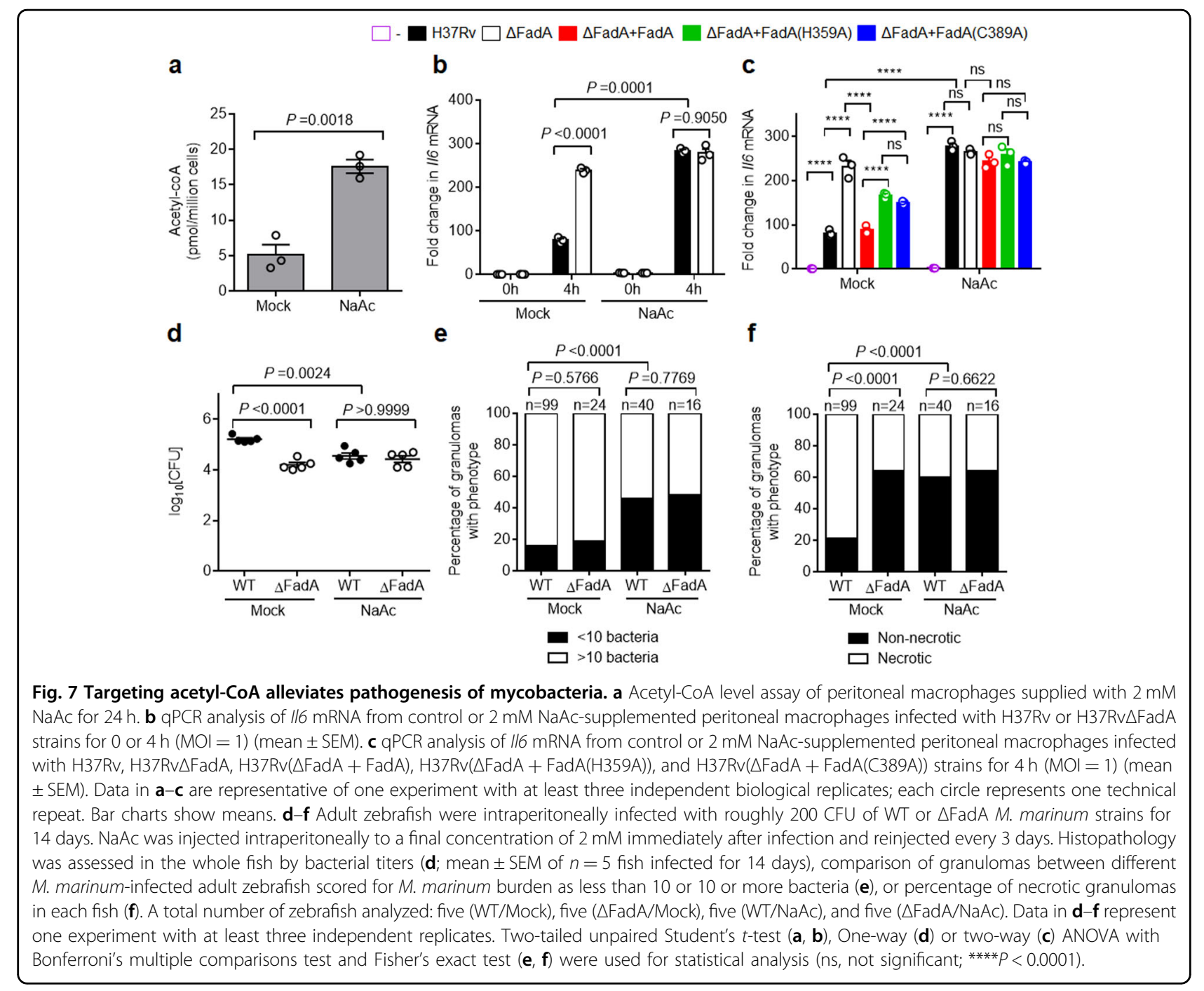




\section{Discussion}

Adaptation to hypoxia, evasion of anti-TB immunity, and utilization of limited host-derived nutrients are major challenges for mycobacteria to establish granulomas and maintain successful persistent infection in the host. In this study, we demonstrate that hypoxia specifically induces FadA, which functions as an immunomodulatory factor to suppress host pro-inflammatory responses. Deletion of FadA attenuates the survival of mycobacteria in vivo through enhancing the host pro-inflammatory responses and preventing granuloma necrosis. FadA-mediated conversion of acetyl-CoA to AcAcCoA may simultaneously provide the substrate for the synthesis of ketone bodies and accumulation of lipid bodies, driving acquisition of the foamy phenotype by host macrophages ${ }^{40-42}$. Thus, FadA appears to be induced by hypoxia to suppress host anti-TB immunity and possibly enhance the accumulation of host-derived nutrients, thus economically facilitating the adaptation of mycobacteria to hypoxic granuloma for persistent infection.

One unusual feature of pathogenic mycobacteria is their five paralogous ESX-1 type VII secretion systems, which transport their effector proteins across the hydrophobic and highly impermeable cell walls into the cytoplasm of host cells ${ }^{10}$. We have profiled the secretion of H37Rv under hypoxia and found that hypoxia either increased or decreased the secretion of different H37Rv proteins, indicating the adaptation of this species to hypoxia through trimming transportation via secretion systems. Interestingly, most proteins that are secreted more by hypoxia are related to fatty acid metabolism, encoded by genes that are already relatively "overrepresented" in the M. tuberculosis genome ${ }^{20}$. The H37Rv protein with the most significant increase in the secretion, FadA, acts as an acetyltransferase that interferes with the host fatty acid metabolism. The function of FadA to convert host acetylCoA to AcAcCoA represents an unusual mechanism in host-microbe interactions, and research may expand to other fatty acid metabolism-related H37Rv secreted proteins including Rv0860 and Rv3774, which are highly expressed under hypoxia.

Pathogenic mycobacteria have evolved to interfere with host innate immune responses to establish successful infection $^{46-48}$. From qRT-PCR validation, we confirmed that FadA could inhibit the expression of the proinflammatory cytokine Il6. It has been shown that IL-6 has a crucial role in protecting against infection by high doses of intravenously delivered M. tuberculosis ${ }^{26}$, but is not required for the generation of specific immunity to infection by low-dose aerosol-delivered $M$. tuberculosis in C57BL/6J mice ${ }^{49}$. Here, our study indicates that the inhibition of IL- 6 by genetic deletion or antibody neutralization promotes the progression of granuloma as well as the survival of mycobacteria in adult zebrafish infected with $M$. marinum or $\mathrm{C} 3 \mathrm{HeB} / \mathrm{FeJ}$ mice infected with H37Rv. To our knowledge, this is the first study to demonstrate an inhibitory role of IL- 6 in the progression of mycobacterial granuloma. Furthermore, our data show that FadA promotes the survival of mycobacteria and the progression of tuberculous granuloma necrosis through inhibiting IL-6. M. tuberculosis infection usually activates the MAP kinase or NF- $\mathrm{kB}$ pathway to induce the expression of proinflammatory cytokines such as $I l 6^{46-48}$. However, activation of the MAP kinase or NF- $\mathrm{kB}$ pathway is not inhibited by FadA (data not shown). Histone modifications such as hyperacetylation of histone $\mathrm{H} 3$ are known to be active markers of gene expression and are often associated with the ongoing transcription of cytokines $^{29,50}$. Our data demonstrate that FadA inhibits the acetylation of $\mathrm{H} 3 \mathrm{~K} 9$ at the $\mathrm{Il6}$ promoter of $\mathrm{H} 37 \mathrm{Rv}-$ infected macrophages, and suppresses the expression of the Il6 gene through modulating histone acetylation, suggesting a previously unidentified but important role of histone acetylation in the regulation of $M$. tuberculosisinduced proinflammatory responses. The histone code hypothesis suggests that multiple histone modifications act in a combinatorial fashion to specify distinct chromatin states ${ }^{51}$. Therefore, more acetylation positions of histones in macrophages infected with the $H 37 \mathrm{Rv}$ or $\mathrm{H} 37 \mathrm{Rv} \Delta \mathrm{FadA}$ strains need to be detected and analyzed together.

Most mycobacterial secreted proteins are believed to inhibit host innate immune responses through interacting with host immune signaling proteins ${ }^{46-48,52}$. However, recent studies have indicated that the metabolic regulation of host macrophages determines pathogen growth or containment ${ }^{53-55}$. Successful import and utilization of host-derived fatty acids are required for the physiology and pathogenesis of $M$. tuberculosis ${ }^{21,56,57}$. Here, we found that FadA acted as an acetyl-CoA acetyltransferase to reduce the cytoplasmic level of acetyl-CoA. Acetyl$\mathrm{CoA}$, as a substrate for histone acetylation ${ }^{35,36}$, has been found to increase histone H3K9 acetylation to trigger the expression of proinflammatory cytokines in $\mathrm{H} 37 \mathrm{Rv}$ infected macrophages. It has been reported that the virulent H37Rv protein ESAT-6 modulates mitochondrial pyruvate transporter activity to markedly increase the total cellular level of acetyl-CoA ${ }^{40-42}$. However, the excessive accumulation of acetyl-CoA may facilitate histone acetylation to increase the expression of proinflammatory cytokines and promote an effective immune response against mycobacteria. Simultaneously, FadA appears to counteract the ESAT-6-mediated increase in cytoplasmic acetyl-CoA to suppress histone acetylationmediated anti-TB immunity. We further speculate that the production of AcAcCoA by FadA may simultaneously promote the production of AcAcCoA as the substrate for the synthesis of ketone bodies and accumulation of lipid 
bodies, driving acquisition of the foamy phenotype by the host macrophages ${ }^{40,42}$. However, the exact mechanism underlying the regulation of FadA in lipid synthesis requires further study.

There is an urgent need to develop novel therapeutic approaches for the clinical treatment of $\mathrm{TB}$, especially drug-resistant TB. HDT is emerging as an attractive strategy harnessing the host-microbe interaction to generate immune responses against $M$. tuberculosis instead of direct bactericidal effects ${ }^{44}$. Here, we demonstrate that acetate supplementation can increase the level of acetyl$\mathrm{CoA}$ in peritoneal macrophages, and markedly reduce granuloma necrosis and mycobacterial burden in the adult zebrafish- $M$. marinum infection model. In addition, the three-dimensional structure of FadA has already been solved $^{37}$, and two evolutionarily conserved enzymatic sites, H359 and C389, have been found to be essential for virulence. Using computer-assisted simulation, we have identified 80 compounds acting against the activity of FadA, and study of the effects of these compounds on the survival of mycobacteria and granuloma formation is currently ongoing. Together, our findings provide a novel HDT strategy for TB infection by targeting the interaction of FadA with the host fatty acid metabolic pathway.

In summary, our findings identify FadA as a previously unrecognized mycobacterial virulence factor that harnesses an unusual mechanism of integrating the adaptation to hypoxia with the inhibition of host anti-TB immunity and probably utilizes host-derived nutrients to facilitate the formation of the granuloma for persistent infection (Supplementary Fig. S12). The reduction in the host acetyl-CoA level by FadA to suppress anti-TB immunity is distinguished from the utilization of hostderived fatty acids as favored nutrient resources for $M$. tuberculosis ${ }^{54,55,57}$. Accordingly, the conversion of acetylCoA to AcAcCoA by FadA reduces the cytoplasmic level of acetyl-CoA in host cells to suppress the histone H3K9 acetylation-mediated expression of $I l 6$, a factor not previously known to inhibit granuloma pathology. Therefore, the depletion of host acetyl-CoA by hypoxia-induced FadA represents an unusual strategy for $M$. tuberculosis under hypoxic conditions to intercept the host fatty acid metabolism to suppress anti-TB immunity and adapt to hypoxic granuloma for persistent infection. This provides insights for the design of effective approaches to eliminate TB or even drug-resistant TB infection through targeting the interface between mycobacteria and host fatty acid metabolism.

\section{Materials and methods \\ Bacterial strains and cells}

All strains used in this study are described in Supplementary Table S3. The Mycobacterium tuberculosis H37Rv, Mycobacterium marinum Aronson (BAA-535) strains were grown in Middlebrook 7H9 broth (Difco/ Becton Dickson, Franklin Lakes, NJ) supplemented with $10 \%$ ADC (5\% bovine serum albumin (BSA), 2\% dextrose, $5 \%$ catalase) and $0.05 \%$ Tween- 80 (Sigma) or Middlebrook 7H10 agar (Difco) supplemented with 10\% ADC and antibiotic supplements as required. The antibiotics used were $50 \mu \mathrm{g} / \mathrm{mL}$ or $75 \mu \mathrm{g} / \mathrm{mL}$ hygromycin B (Thermo) and $50 \mu \mathrm{g} / \mathrm{mL}$ kanamycin (Sigma).

Primary peritoneal macrophages were harvested as described previously ${ }^{58}$. Briefly, C57Bl/6 mice were intraperitoneally injected with $2 \mathrm{~mL}$ of $4 \%$ Brewer thioglycollate medium (Sigma). After 3 days, the mice were sacrificed by cervical dislocation, and cells were isolated by flushing the peritoneal cavity with $10 \mathrm{~mL}$ of RPMI1640 (Thermo) medium per mouse. Cells were seeded in a 24-well dish, and non-adherent cells were removed by extensive washing with RPMI-1640. The adherent peritoneal macrophages were cultured in RPMI-1640 medium supplemented with $10 \%(\mathrm{v} / \mathrm{v})$ heat-inactivated fetal bovine serum (FBS, Thermo) and $100 \mathrm{U} / \mathrm{mL}$ penicillin and streptomycin (Thermo) and used for subsequent experiments. All the cells were routinely tested for contamination by mycoplasma.

\section{Bacterial cultures}

Wayne experiments in $\mathrm{H} 37 \mathrm{Rv}$ were performed as described previously ${ }^{18}$. In brief, conical screw-capped Nephelo flasks with $20 \mathrm{~mm}$ side arms and flat bases (Wheaton Scientific Products, Millville, NJ) were used to culture bacteria. H37Rv was grown to mid-log phase $\left(\mathrm{OD} 590 \approx 0.4\right.$, probably $2.5 \times 10^{8}$ colony-forming unit $(\mathrm{CFU}) / \mathrm{mL}$ ). For aerobic conditions, $200 \mathrm{~mL}$ of medium was inoculated with $2 \mathrm{~mL}$ of the culture and incubated at $37^{\circ} \mathrm{C}$ on a magnetic stirrer set rotating at $180 \mathrm{rpm}$. Simultaneously, $400 \mathrm{~mL}$ of medium was inoculated with $4 \mathrm{~mL}$ of identical culture in a tightly capped flask, placed on a tissue culture magnetic stirrer set at $70 \mathrm{rpm}$, and incubated at $37^{\circ} \mathrm{C}$ to avoid perturbation of the surface. Samples for the quantitative proteomic assay were taken after 14 days of vigorous aeration of cultures (aeration) and hypoxic cultures (hypoxia).

For western blotting or qPCR detection of bacterial cultures, mycobacteria were grown to the mid-log phase as before. For aerobic conditions, $4 \mathrm{~mL}$ of the protein-free liquid medium in the $20 \mathrm{~mm}$ screw-capped culture tubes were inoculated with $0.4 \mathrm{~mL}$ of the culture and incubated at $37^{\circ} \mathrm{C}$ in a shaker-incubator set rotating at $180 \mathrm{rpm}$. Simultaneously, $8 \mathrm{~mL}$ of medium was inoculated with $0.8 \mathrm{~mL}$ of identical culture in the tightly capped tubes, placed on a shaker-incubator set rotating at $70 \mathrm{rpm}$, and incubated at $37^{\circ} \mathrm{C}$ to avoid perturbation of the surface. Then $4 \mathrm{~mL}$ cultures under aeration or hypoxia after indicated days were individually collected and centrifuged to separate the culture supernatant and precipitated 
bacteria cell. For samples at day 0 , identical mycobacteria cultures were added in the aeration or hypoxia culture tubes similarly, and the culture supernatant and precipitated bacteria cell were collected immediately.

$\mathrm{H} 37 \mathrm{Rv}$ and M. marinum cultures were grown up to the mid-log phase. Aliquots of bacterial cultures were prepared in 20\% glycerol and Middlebrook 7H9 medium and preserved at $-80^{\circ} \mathrm{C}$. The CFU per milliliter of the stocks was titered by plating serial dilutions on Middlebrook 7H10 agar plates plus 10\% ADC. These stocks were used for all subsequent infections of macrophages, zebrafish, and mice.

\section{Plasmids, reagents, and antibodies}

Plasmids used in this study are described in Supplementary Table S3. Direct-Blot HRP anti-E. coli RNA Sigma 70 (SigA) antibody (663205) was purchased from BioLegend. Rabbit polyclonal to ESAT6 (ab45073) and polyclonal rabbit anti-Histone H3 (acetyl K9) antibody (ab4441, ChIP grade) were purchased from Abcam. The rabbit polyclonal antibody to FadA was generated by immunization of rabbits with the purified SUMO-FadA fusion protein, in collaboration with ABclonal Biotech, so as the isotype control antibody. InVivo Mab anti-mouse IL-6 (BE0046) and InVivo Mab rat IgG1 isotype control (BE0088) were purchased from BioXcell. Recombinant ESAT6 protein was from the laboratory stock. Enzymatic chromatin IP kit (magnetic beads) (9003) was from Cell Signaling Technology. Acetyl-Coenzyme A Assay Kit (MAK039) was from Sigma. ECL reagent (34075) was from Thermo.

\section{Quantitative comparative proteomic profiling of culture filtrate supernatant from H37Rv under aeration or hypoxia}

The culture supernatant and collected cells were separated via filtration through a membrane with $0.22 \mu \mathrm{m}$ pore (Millipore). The culture filtrate protein samples were prepared. Briefly, ProteoMiner ${ }^{\mathrm{TM}}$ Protein Enrichment Small-Capacity Kit was used following the manufacturer's instructions. Then, the protein concentration was determined with a 2-D Quant kit, in accordance with the manufacturer's instructions. For trypsin digestion, the supernatant was transferred to a new tube, reduced with DTT for $1 \mathrm{~h}$ at $37^{\circ} \mathrm{C}$, and alkylated with IAA for $45 \mathrm{~min}$ at room temperature in the dark. The protein was precipitated with prechilled acetone overnight at $-20^{\circ} \mathrm{C}$, washed with acetone three times, and then redissolved in TEAB. Approximately, $200 \mu \mathrm{g}$ of protein for each sample was digested with trypsin overnight at $37^{\circ} \mathrm{C}$. After trypsin digestion, the peptide was desalted using a Strata X C18 SPE column (Phenomenex) and vacuum-dried. The peptide was reconstituted in TEAB and processed in accordance with the manufacturer's protocol for the 6-plex TMT kit. Peptides were dissolved, directly loaded onto a reversed-phase column packed in-house with $3-\mu \mathrm{m} \mathrm{C18}$ beads (Reprosil-Pur C18-AQ, Dr. Maisch), and eluted with a linear gradient of solvent B on an EASY-nLC 1000 UPLC system. The resulting peptides were analyzed by Q Exactive (ThermoFisher) and subjected to NSI source, followed by tandem mass spectrometry (MS/MS) coupled online with UPLC. For MS scans, the $\mathrm{m} / \mathrm{z}$ scan range was 350-1600 Da. The MS/MS data files were merged and transformed to an MGF file by using Proteome Discoverer (Version 1.3.0.339, Thermo). Peptide and protein identifications were performed using the Mascot search engine (Version 3.2). We collected the protein sequence of H37Rv from Uniprot. The complete list of identified peptides was then housed in an Excel file for the grouping of results into proteins and the calculation of ratios and coefficients of variation.

\section{Immunolocalization analysis}

For immunohistochemistry (IHC), lung tissue from a TB patient or a lung cancer patient with pulmonary lobectomy and whole fish sections of $M$. marinum infected or uninfected adult zebrafish were investigated. Segments of lung tissues and whole fish were fixed in $10 \%$ buffered formalin and embedded in paraffin. The blocks were cut into $5 \mu \mathrm{m}$ sections, and five noncontiguous sections were processed for IHC staining with anti-FadA polyclonal antibody at a 1:100 dilution and anti-rabbit secondary antibody labeled with HRP (Servicebio) at a 1:200 dilution, compared with antiESAT-6 polyclonal antibody at a 1:200 dilution, isotype polyclonal control antibody at a 1:100 dilution, anti-SigA antibody labeled with HRP at 1:100 dilution and acid-fast staining. Immunostaining was visualized with 3,3-diaminobenzidine (DAB, Servicebio) substrate. After counterstaining in hematoxylin, the sections were mounted and examined. The studies on clinical samples were conducted in accordance with ethical guidelines of the Institutional Review Board of Shanghai Pulmonary Hospital (SYXK2018-0033).

\section{Construction of fadA-modified mycobacterial strains}

Gene knockouts of FadA in H37Rv and M. marinum were generated using allelic exchange and a specialized transducing phage, phAE87, as described previously ${ }^{59}$. In brief, upstream and downstream flanking regions were amplified using the primer pairs and cloned into the delivery vector pYUB854. Allelic exchange constructs were incorporated into the phAE87 phage and phagemid DNA was electroporated into electrocompetent $M$. smegmatis cells to obtain plaques from the transduced 7H10 agar plates. Specialized transducing phages were picked and amplified at $30^{\circ} \mathrm{C}$ to generate high-titer mycobacteriophages. The desired phage was transduced into H37Rv or M. marinum to delete the FadA gene by 
specialized transduction. The transductants were plated on selective medium, 7H10 agar containing 10\% ADC enrichment and $75 \mu \mathrm{g} / \mathrm{mL}$ hygromycin, and cultured at $37^{\circ} \mathrm{C}$ for $\mathrm{H} 37 \mathrm{Rv}, 30^{\circ} \mathrm{C}$ for $M$. marinum. Hygromycinresistant clones were isolated and analyzed by PCR and qPCR to confirm the deletion of target genes. Western blotting was used to confirm the deletion of FadA with anti-FadA polyclonal antibody at a 1:1000 dilution; antiSigA monoclonal antibody at a 1:1000 dilution was used as the reference antibody. The complementation plasmid for the FadA mutant was generated by ligating the PCR products using FadA-specific primers (Supplementary Table S4) into the episomal vector, pVV16 with hsp60 promoter. The resulting plasmid was transformed into the $\Delta$ FadA strain and plated on a $7 \mathrm{H} 10$ plate containing kanamycin at $50 \mu \mathrm{g} / \mathrm{mL}$. Positive integrants carrying the required insert were screened by colony PCR and validated by $\mathrm{qPCR}$ and immunoblot analyses.

\section{Growth curve determination}

Mycobacteria were grown to mid-log phase in $7 \mathrm{H} 9$ broth with $10 \%$ ADC, $0.05 \%$ Tween 80 , and antibiotics, as required. Growth curves for each strain were determined using a Bioscreen $\mathrm{C}$ Growth Curve Instrument (Labsystems Oy, Helsinki, Finland) and a honeycomb plate with 100 wells (Labsystems Oy). Briefly, $200 \mu \mathrm{L}$ of each bacterial suspension, adjusted to a similar density, was added to each well and cultured with shaking at $37^{\circ} \mathrm{C}$ for H37Rv and $30^{\circ} \mathrm{C}$ for M. marinum. The optical density was measured at an absorbance of $590 \mathrm{~nm}$ every day. Hypoxic conditions were established by covering each culture with $50 \mu \mathrm{L}$ of paraffin oil. Cultures were incubated at $37^{\circ} \mathrm{C}$ for 14 days for $\mathrm{H} 37 \mathrm{Rv}$ and 10 days for M. marinum. Three independent experiments were performed, each in triplicate.

\section{ChIP-seq and ChIP-qPCR analysis}

ChIP was were performed with SimpleChIP Enzymatic Chromatin IP Kit, in accordance with the manufacturer's instructions. In brief, primary peritoneal macrophages were stimulated with $\mathrm{H} 37 \mathrm{Rv}$ or H37Rv $\Delta$ FadA strains for $4 \mathrm{~h}$ and were fixed for $10 \mathrm{~min}$ at $25^{\circ} \mathrm{C}$ with $1 \%$ formaldehyde. After incubation, glycine was added to a final concentration of $0.125 \mathrm{M}$ to quench formaldehyde. Subsequently, cells were lysed and chromatin was harvested and fragmented using enzymatic digestion followed by sonication. The chromatin was then subjected to immunoprecipitation with anti$\mathrm{H} 3 \mathrm{~K} 9 \mathrm{Ac}$ at $4{ }^{\circ} \mathrm{C}$ overnight and was incubated with protein $\mathrm{G}$ magnetic beads at $4{ }^{\circ} \mathrm{C}$ for $2 \mathrm{~h}$. The immune complexes were washed and eluted in $150 \mu \mathrm{L}$ of elution buffer. Elute DNA and input DNA were incubated at $65^{\circ} \mathrm{C}$ to reverse the cross-linking. After digestion with proteinase $\mathrm{K}$, DNA was purified with spin columns.
The relative abundance of precipitated DNA fragments was analyzed by qPCR using SYBR Green PCR Master Mix and the enrichments were normalized to Rpl30 promoter (Cell Signaling Technology). Primer pairs used for Il6 ChIP-qPCR are listed in Supplementary Table S4.

\section{ChIP-seq data analysis}

ChIP sample quality was assessed using a bioanalyzer (Agilent Technologies). ChIP-seq libraries were prepared in accordance with the Illumina TruSeq ChIP sample protocol. They were then pooled for deep sequencing using Illumina Hiseq Xten $(2 \times 150)$ platforms at the CASMPG Partner Institute for Computational Biology Omics Core (Shanghai, China). Raw read quality was evaluated with FastQC (http://www.bioinformatics.babraham.ac.uk/ projects/fastqc/v0.11.5). Adapter sequences and read sequences on both ends with Phred quality scores below 30 were trimmed. Trimmed reads were then mapped with the Bowtie2 algorithm (version 2.3.0) to the mouse genome (version mm10). Reads that failed to be mapped or that mapped at several locations (as identified by the XS tag set by Bowtie2) were removed. Read duplicates were identified and removed using Picard's Mark Duplicates (http://broadinstitute.github.io/picard). Peak calling was performed using MACS2 (version 2.1.1). For all experiments, MACS2-defined peaks from all conditions (-, H37Rv and $\triangle \mathrm{FadA})$ were merged into a unique nonoverlapping set of 21,708 peaks for H3K9AcIP (filtered for $P$ value $<0.00001)$. Genome-wide signal coverage tracks at every bp were also computed by MACS2 and visualized in the UCSC Genome Browser. Peaks were annotated to the nearest genes within $2 \mathrm{~kb}$ of the TSS using ngsplot (version 2.61). Peaks overlapping by at least 1 nt with unique gene model promoters $( \pm 2 \mathrm{~kb}$ of each unique gene model transcription starting site) were considered as being located at promoters. De novo motif searches of ChIP-seq peaks were performed using MEME.

\section{Purification of FadA and its derivative mutants}

The construction and purification of H37Rv FadA recombinant protein and its derivative mutants were performed as described previously. pET28a-FadA, pET28a-FadA(H359A), and pET28a-FadA(C389A) plasmids are described in Supplementary Table S3.

\section{In vitro two-substrate steady-state kinetics assay of acetyltransferase activity}

Acetyltransferase activity was measured following the methods of Thompson et al. ${ }^{60}$, and Middleton ${ }^{61}$. In brief, assay mixtures containing $100 \mathrm{mM}$ Tris- $\mathrm{HCl}, \mathrm{pH}$ 8.1, $25 \mathrm{mM} \mathrm{MgCl} 2,50 \mathrm{mM}-\mathrm{KCI}$, and $0-200 \mathrm{M}$ acetyl-CoA (Sigma) were preincubated at $30^{\circ} \mathrm{C}$ for $5 \mathrm{~min}$. The background rate of acetyl transfer was measured. Reactions were 
initiated by the addition of FadA or its derivative mutants $(250 \mathrm{nM})$ to the assay mixture. The formation of acetoacetyl-CoA- $\mathrm{Mg}^{2}$-enolate was followed at $303 \mathrm{~nm}$ for $5 \mathrm{~min}$ in scanning mode using a Varioskan Flash MultiMode Microplate Reader (Thermo) and its associated software, and the initial velocity was determined from the linear portion, at approximately $2 \mathrm{~min}$. The background rate of thiolysis in the absence of FadA was subtracted from the measured enzymatic rate.

\section{Acetyl-CoA level assay of peritoneal macrophages}

For the measurement of cytosolic acetyl-CoA, primary peritoneal macrophages were stimulated with different strains or components for $4 \mathrm{~h}$ and were lysed with lysis buffer (1\% Triton X-100, $20 \mathrm{mM}$ Tris-HCl, $\mathrm{pH} 7.4,150 \mathrm{mM}$ $\mathrm{NaCl}$ ) on ice for $10 \mathrm{~min}$. The lysates were spun at $20,000 \times \mathrm{g}$ for $10 \mathrm{~min}$ at $4{ }^{\circ} \mathrm{C}$, the pellets were discarded, and the supernatants were used for acetyl-CoA measurement with an Acetyl-CoA Assay Kit (Sigma). Freshly isolated primary peritoneal macrophages were rested in RPMI-1640 medium with $2 \mathrm{mM}$ sodium acetate (NaAc) for $24 \mathrm{~h}$ at $37^{\circ} \mathrm{C}$ in an incubator, and the level of acetyl-CoA was measured.

\section{qRT-PCR analysis}

RNA preparation and $\mathrm{qPCR}$ analysis were performed as described previously using gene-specific primers (Supplementary Table S4). Total RNA was extracted with $1 \mathrm{~mL}$ of Trizol reagent in accordance with the instructions of the manufacturer (ThermoFisher). One microgram of total RNA was applied for cDNA synthesis using the ReverTra Ace ${ }^{\circledR}$ qPCR RT Kit (Toyobo). Real-time quantitative PCR was performed using the SYBR RT-PCR Kit (Toyobo) in an LC480 thermocycler (Roche, Indianapolis, IN). The $2^{-\Delta \Delta C t}$ method was adopted to analyze the relative gene expression; the gene expression was normalized to the expression of gapdh for eukaryotic cells and 16sRNA for prokaryotic cells. Real-time qPCR data were collected from at least three independent experiments, with three technical replicates per experiment.

\section{Western blotting}

Standard western blotting procedures were used. Cell extracts or culture filtrates $(50 \mu \mathrm{g})$ of mycobacteria were denatured in $1 \times$ sodium dodecyl sulfate (SDS) protein sample buffer and separated on a $10 \%$ or $12 \%$ SDSpolyacrylamide gel. They were then transferred to nitrocellulose membranes. Next, the membrane was blocked, incubated with primary antibodies, and washed three times before incubation with secondary antibodies. The concentrations of the primary antibodies were 1:1000 for antiFadA, anti-ESAT-6, and anti-SigA antibodies. Horseradish peroxidase-conjugated goat anti-rabbit polyclonal antibody was used as the secondary antibody at a 1:5000 dilution. After a final wash, analysis was conducted using an ECL reagent (Thermo). Densitometric analysis of band intensity was performed using Image J software.

\section{Mice and infection}

Animal procedures were approved by the Animal Experiment Administration Committee of Shanghai Pulmonary Hospital (K18-033), and this study was carried out in strict accordance with the China National Research Council's Guide for Care and Use of Laboratory Animals. All surgeries were performed under sodium pentobarbital anesthesia, and all efforts were made to minimize suffering. Female SPF C57BL/6 mice were purchased from Shanghai SLAC Laboratory Animal Co., Ltd. (Shanghai, China). Female $\mathrm{C} 3 \mathrm{HeB} / \mathrm{FeJ}$ mice were purchased from Jackson Laboratory (Bar Harbor, ME). Totally, 6-8-weekold female C57BL/6 mice were used for macrophage separation. For in vitro $\mathrm{H} 37 \mathrm{Rv}$ infection, mouse macrophages were infected with a single cell suspension of bacteria at a MOI of 1 . Female $\mathrm{C} 3 \mathrm{HeB} / \mathrm{FeJ}$ mice (6-8 weeks old) were infected by an aerosol method with approximately $200 \mathrm{CFU}$ per mice of the indicated bacterial strains (using a Glas-Col inhalation exposure system (Glas-col, Terre Haute, IN) $^{62}$ at an Animal Biosafety Level-3 (ABSL-3) Laboratory. At 1-week post-infection, mice received $0.3 \mathrm{mg}$ of anti-IL-6 mAb (BioXcell) or isotype-matched control Ab (rat IgG1) intranasally once a week for up to 4 weeks. Mice were sacrificed 1 day or 4 weeks after infection. Tissues from the left lung from infected mice were harvested and homogenized in $1 \mathrm{~mL}$ of PBS. Homogenates in 10-fold serial dilutions were plated on 7H10 agar supplemented with 10\% ADC enrichment and incubated at $37^{\circ} \mathrm{C}$. Colonies were counted after 4 weeks of incubation at $37^{\circ} \mathrm{C}$ in $5 \% \mathrm{CO}_{2}$. For histological analysis, half of each lung from infected mice for 4 weeks was fixed in $4 \%$ neutral-buffered paraformaldehyde solution for $24 \mathrm{~h}$ and then embedded in paraffin. A series of sections with a thickness of 4-7 $\mu \mathrm{m}$ were then cut and stained with H\&E or Ziehl-Neelsen (acid-fast bacillus) stain, in accordance with standard protocols. Imaging was performed by microscopy (TCS CP5II; Leica, Germany). The pathology was evaluated by pathologists in a blinded manner. All mice were age-, weight-, and sex-matched in each experiment. The sample size was based on empirical data from pilot experiments. No additional randomization or blinding was used to allocate experimental groups.

\section{Generation of IL-6 knockout zebrafish using CRISPR/Cas9 mutagenesis}

To study the function of IL- 6 in granuloma development, we employed the Cas9/gRNA system to generate IL-6 knockout zebrafish. $i l 6^{12 \mathrm{~d} 2 \mathrm{i}}$ and $i l 6^{20 \mathrm{dl}}$ mutant zebrafish were constructed at the China Zebrafish Resource Center (CZRC), as described previously ${ }^{63}$. In brief, a functional gRNA targeting the second exon of the il6 gene 
was designed and microinjected with Cas9 mRNA into one-cell embryos of zebrafish. The $\mathrm{F}_{0}$-generation fish grew to adulthood. Individual outcrosses of the $\mathrm{F}_{0}$-zebrafish with the WT $\mathrm{AB}$ fish allowed us to screen for germline-transmitted mutations and to identify mutations of interest in the $F_{1}$-progeny. The $F_{1}$-progeny were screened from the sequencing results of the tailfin DNA from the adult zebrafish. The following primers were used for sequencing: (F) 5'-CAGTGCTATTCCTGTCTGCT AC- $3^{\prime}$ and (R) 5'-TAACTGGGTTACTCGTTTTGAGT$3^{\prime}$. The $F_{1}$-zebrafish carrying individual mutations of interest were spawned together to obtain $\mathrm{F}_{2}$-generation progeny for the experiments. Finally, a total of two zebrafish lines bearing different il6 mutations resulting in premature stop codons after 65 and 70 amino acids, were used in the study. These two different il6 null mutant zebrafish lines were named $i l 6^{12 \mathrm{~d} 2 \mathrm{i}}(-10 \mathrm{bp}$, loss of CTGTACAAGGAC and insert of GA) and $i l 6^{20 \mathrm{dl}}$ ( $-20 \mathrm{bp}$, loss of CTGTACAAGGACGTGAAGAC). qPCR was used to confirm the deletion of il6.

\section{Zebrafish infection}

WT zebrafish (Danio rerio) were obtained from CZRC (Wuhan) and husbandry was performed as described previously by Swaim et al. ${ }^{64}$. Briefly, the fish were reared in recirculating fish systems obtained from Qingdao Elvin Marine Technology Co., Ltd. (Qingdao, China), and transferred to a flowthrough fish system for the infection experiment. Up to 10 fish were kept in a $3 \mathrm{~L}$ tank and tanks were maintained under standard conditions for housing zebrafish (water temperature $\sim 28^{\circ} \mathrm{C}, \mathrm{pH} \sim 7.4$, and conductivity $\sim 1500 \mu \mathrm{S})$. Infection protocols approved by the Animal Experiment Administration Committee were used for the zebrafish infection studies. Healthy adult fish were infected as described previously ${ }^{64}$. Fish were infected by intraperitoneal injection with approximately $200 \mathrm{CFU}$ of the indicated bacterial strains after first anesthetizing with $0.1 \%$ 3-aminobenzoic acid ethyl ester (tricaine; Sigma). Bacterial CFU contained in the injected inoculum was confirmed by plating onto $7 \mathrm{H} 10$ agar. To assess the bacterial burden from whole fish post-infection, the fish infected for 1 or 14 days were terminally anesthetized in $0.5 \%$ tricaine, homogenized in $1.0 \mathrm{~mL}$ of $\mathrm{PBS}$, and then the dilutions were plated on 7H10 agar plates. Three or five fish were plated for each condition and the resulting bacterial counts were determined. For histological analysis, fish infected for 14 days were fixed in $4 \%$ neutral-buffered paraformaldehyde solution for $72 \mathrm{~h}$, embedded in paraffin, sectioned, and stained with $\mathrm{H} \& \mathrm{E}$ or Ziehl-Neelsen stain. According to the methods described previously ${ }^{15,65}$, acid-fast staining of granulomas demonstrates the presence of acid-fast bacilli and bacterial burden in the granulomas, H\&E staining indicates the necrotic regions of granulomas. Granulomas of different $M$. marinum-infected adult zebrafish scored for
M. marinum burden as less than 10 or 10 or more bacteria and the percentage of necrotic granulomas was quantified and compared based on the staining results. A total number of granulomas for each strain was counted and shown with " $n$ ", and the different number of granulomas between different groups also reflected the pathology of M. marinuminfected zebrafish.

\section{Statistical analysis}

Data from independent experiments were expressed as the mean \pm SEM. All experimental data were analyzed using GraphPad Prism software. The statistical significance of differences between the two groups was determined by a two-tailed unpaired Student's $t$-test. One-way or two-way ANOVA with Bonferroni's multiple comparisons test was used for statistical analysis when comparing more than two groups. Populations were compared by Fisher's exact test. Differences were considered significant at $P<0.05$.

\section{Acknowledgements}

We thank Dr. Liang-Dong Lyu (CAS Key Laboratory of Synthetic Biology, Shanghai, China) for technical assistance to FadA knockout strain construction. We thank Profs. Feng Shao (National Institute of Biological Sciences, Beijing, China), Carl G. Feng (Sydney Medical School, the University of Sydney, Sydney, NSW, Australia), and Zhiming Zhao (Fudan University, Shanghai, China) for critical reading of the paper. We thank members of the B. Ge laboratory for helpful discussions and technical assistance. This project was supported by grants from the National Natural Science Foundation of China (81870002 and 82070007 to H.Y. and $9184230036,31730025,91542111,81330069,31030028$ and 30525012 to B.G.), the Chinese National Program on Key Basic Research Project (2017YFA0505900 to B.G.), the Chinese National Key Project for Infectious Disease (2018ZX10722302-004 to H.Y.) and the most important clinical discipline in Shanghai (2017ZZ02003 to B.G.).

\section{Author details}

'Shanghai Key Laboratory of Tuberculosis, Shanghai Pulmonary Hospital, Tongji University School of Medicine, Shanghai, China. ${ }^{2}$ Department of Microbiology and Immunology, Tongji University School of Medicine, Shanghai, China. ${ }^{3}$ Tuberculosis Center for Diagnosis and Treatment, Shanghai Pulmonary Hospital, Tongji University School of Medicine, Shanghai, China. ${ }^{4}$ State Key Laboratory of Genetic Engineering, Institute of Genetics, School of Life Science, Fudan University, Shanghai, China. ${ }^{5}$ Department of Pathology, Shanghai Pulmonary Hospital, Tongji University School of Medicine, Shanghai, China. ${ }^{6}$ Tuberculosis Research Program at the Centenary Institute, The University of Sydney, Sydney, NSW, Australia. 'Eastern China Center for Pathogen Discovery and Research, Shanghai Public Health Clinical Center, Fudan University, Shanghai, China. ${ }^{8}$ Omics Core of Bio-Med Big Data Center, CAS-MPG Partner Institute for Computational Biology, Shanghai Institutes for Biological Sciences, Chinese Academy of Sciences, Shanghai, China. ${ }^{9}$ China Zebrafish Resource Center, Institute of Hydrobiology, Chinese Academy of Sciences, Wuhan, Hubei, China

\section{Author contributions}

H.Y., F.L., and B.G. designed this study, assisted by H.L. H.Y., F.W., X.G., Z.L., X.W. M.Z., M.M., L.Q., T.T., Y.W., X.H., and J.W. performed most experiments and analyzed data, assisted by L.W., W.S., J.C., C.P., R.Z., F.T., and L.Z. H.Y. and J.W. constructed recombinant H37Rv and M. marinum strains. F.W., X.G., M.Z., Y.W., and C.P. performed zebrafish infection experiments, supported by L.Z. and S.H.O. Immunolocalization of lung granuloma sections were supported by C.W. Mice infection was supported by Z.S. and J.S. ChIP-seq data analysis was supported by H.F. All the mycobacteria strains were conserved and subcultured by X.H. IL-6 knockout zebrafish was created by X.X. All authors discussed the results and commented on the paper. 


\section{Conflict of interest}

The authors declare no competing interests.

\section{Publisher's note}

Springer Nature remains neutral with regard to jurisdictional claims in published maps and institutional affiliations.

Supplementary information The online version contains supplementary material available at https://doi.org/10.1038/s41421-021-00301-1.

Received: 9 May 2021 Accepted: 1 July 2021

Published online: 05 October 2021

\section{References}

1. World Health Organization. Global tuberculosis report 2020. (World Health Organization, Geneva, Licence: CC BY-NC-SA 3.0 IGO, 2020).

2. Pagán, A. J. \& Ramakrishnan, L. The formation and function of granulomas. Annu. Rev. Immunol. 36, 639-665 (2018).

3. Davis, J. M. \& Ramakrishnan, L. The role of the granuloma in expansion and dissemination of early tuberculous infection. Cell 136, 37-49 (2009).

4. Chao, M. C. \& Rubin, E. J. Letting sleeping dos lie: does dormancy play a role in tuberculosis? Annu. Rev. Microbiol. 64, 293-311 (2010).

5. Via, L. E. et al. Tuberculous granulomas are hypoxic in guinea pigs, rabbits, and nonhuman primates. Infect. Immun. 76, 2333-2240 (2008).

6. Barry, C. E. 3rd et al. The spectrum of latent tuberculosis: rethinking the biology and intervention strategies. Nat. Rev. Microbiol. 7, 845-855 (2009).

7. Rittershaus, E. S., Baek, S. H. \& Sassetti, C. M. The normalcy of dormancy: common themes in microbial. quiescence. Cell Host Microbe 13, 643-651 (2013).

8. Matty, M. A., Roca, F. J., Cronan, M. R. \& Tobin, D. M. Adventures within the speckled band: heterogeneity, angiogenesis, and balanced inflammation in the tuberculous granuloma. Immunol. Rev. 264, 276-287 (2015).

9. Galagan, J. E. et al. The Mycobacterium tuberculosis regulatory network and hypoxia. Nature 499, 178-183 (2013).

10. Gröschel, M. I., Sayes, F., Simeone, R., Majessi, L. \& Brosch, R. ESX secretion systems: mycobacterial evolution to counter host immunity. Nat. Rev. Microbiol. 14, 677-691 (2016).

11. Carow, B. et al. Spatial and temporal localization of immune transcripts defines hallmarks and diversity in the tuberculosis granuloma. Nat. Commun. 10, 1823 (2019).

12. Volkman, H. E. et al. Tuberculous granuloma induction via interaction of a bacterial secreted protein with host epithelium. Science 327, 466-469 (2010).

13. Tobin, D. M. et al. Host genotype-specific therapies can optimize the inflammatory response to mycobacterial infections. Cell 148, 434-446 (2012).

14. Roca, F. J. \& Ramakrishnan, L. TNF dually mediates resistance and susceptibility to mycobacteria via mitochondrial reactive oxygen species. Cell 153, 521-534 (2013)

15. Oehlers, S. H. et al. Interception of host angiogenic signaling limits mycobacterial growth. Nature 517, 612-615 (2015).

16. Walton, E. M. et al. Cyclopropane modification of trehalose dimycolate drives granuloma angiogenesis and mycobacterial growth through vegf signaling. Cell Host Microbe 24, 514-525 (2018).

17. Roca, F. J., Whitworth, L. J., Redmond, S., Jones, A. A. \& Ramakrishnan, L. TNF induces pathogenic programmed macrophage necrosis in tuberculosis through a mitochondrial-lysosomal-endoplasmic reticulum circuit. Cell 178, 1344-1361 (2019).

18. Wayne, L. G. \& Hayes, L. G. An in vitro model for sequential study of shift down of Mycobacterium tuberculosis through two stages of nonreplicating persistence. Infect. Immun. 64, 2062-2069 (1996).

19. Wayne, L. G. \& Sohaskey, C. D. Nonreplicating persistence of Mycobacterium tuberculosis. Annu. Rev. Microbiol. 55, 139-163 (2001).

20. Cole, S. T. et al. Deciphering the biology of Mycobacterium tuberculosis from the complete genome sequence. Nature 393, 537-544 (1998).

21. Lee, W., VanderVen, B. C., Fahey, R. J. \& Russell, D. G. Inracellular Mycobacterium tuberculosis exploits host-derived fatty acids to limit metabolic stress. J. Biol. Chem. 288, 6788-6800 (2013).
22. Stewart, G. R., Robertson, B. D. \& Young, D. B. Tuberculosis: a problem with persistence. Nat. Rev. Microbiol. 1, 97 (2003).

23. Ramakrishnan, L. Revisiting the role of the granuloma in tuberculosis. Nat. Rev. Immunol. 12, 352-366 (2012).

24. Cadena, A. M., Fortune, S. M. \& Flynn, J. L. Heterogeneity in tuberculosis. Nat. Rev. Immunol. 1, 691-702 (2017).

25. Pan, H. et al. Ipr1 gene mediates innate immunity to tuberculosis. Nature $\mathbf{4 3 4}$, 767-772 (2005).

26. Ladel, C. H. et al. Lethal tuberculosis in interleukin-6-deficient mutant mice. Infect. Immun. 65, 4843-4849 (1997).

27. Wang, H. et al. One-step generation of mice carrying mutations in multiple genes by CRISPR/Cas-mediated genome engineering. Cell 153, 910-918 (2013).

28. Kathirvel, M. \& Mahadevan, S. The role of epigenetics in tuberculosis infection. Epigenomics 8, 537-549 (2016).

29. Niller, H. H., Masa, R., Venkei, A., Mészáros, S. \& Minarovits, J. Pathogenic mechanisms of intracellular bacteria. Curr. Opin. Infect. Dis. 30, 309-315 (2017).

30. Yaseen, I., Kaur, P., Nandicoori, V. K. \& Khosla, S. Mycobacteria modulate host epigenetic machinery by Rv 1988 methylation of a non-tail arginine of histone H3. Nat. Commun. 6, 8922 (2015).

31. Moores, R. C., Brilha, S., Schutgens, F., Elkington, P. T. \& Friedland, J. S. Epigenetic regulation of matrix metalloproteinase-1 and -3 expression in Mycobacterium tuberculosis infection. Front. Immunol. 8, 602 (2017).

32. Monneret, C. Histone deacetylase inhibitors. Eur. J. Med. Chem. 40, 1-13 (2005).

33. Parenti, M. D. et al. Discovery of novel and selective SIRT6 inhibitors. J. Med. Chem. 57, 4796-4804 (2014).

34. Esterhuyse, M. M. et al. Epigenetics and proteomics join transcriptomics in the quest for tuberculosis biomarkers. MBio 6, e01187-15 (2015).

35. Sabari, B. R., Zhang, D., Allis, C. D. \& Zhao, Y. Metabolic regulation of gene expression through histone acylations. Nat. Rev. Mol. Cell Biol. 18, 90-101 (2017).

36. Baell, J. B. et al. Inhibitors of histone acetyltransferases KAT6A/B induce senescence and arrest tumour growth. Nature 560, 253-257 (2018).

37. Venkatesan, R. \& Wierenga, R. K. Structure of mycobacterial $\beta$-oxidation trifunctional enzyme reveals its altered assembly and putative substrate channeling pathway. ACS Chem. Biol. 8, 1063-1073 (2013).

38. Jaiswal, A. K., Husaini, S. H. A., Kumar, A. \& Subbarao, N. Designing novel inhibitors against Mycobacterium tuberculosis FadA5 (acetyl-CoA acetyltransferase) by virtual screening of known anti-tuberculosis (bioactive) compounds. Bioinformation 14, 327-336 (2018).

39. Nesbitt, N. M. et al. A thiolase of Mycobacterium tuberculosis is required for virulence and production of androstenedione and androstadienedione from cholesterol. Infect. Immun. 78, 275-282 (2010).

40. Mehrotra, P. et al. Pathogenicity of Mycobacterium tuberculosis is expressed by regulating metabolic thresholds of the host macrophage. PLoS Pathog. 10, e1004265 (2014).

41. Singh, V., Kaur, C., Chaudhary, V. K., Rao, K. V. \& Chatterjee, S. M. tuberculosis secretory protein ESAT-6 induces metabolic flux perturbations to drive foamy macrophage differentiation. Sci. Rep. 5, 12906 (2015).

42. Mehrotra, P., Rao, K. V. \& Chatterjeeb, S. A mathematical model predicting host mitochondrial pyruvate transporter activity to be a critical regulator of Mycobacterium tuberculosis pathogenicity. Biosystems 155, 1-9 (2017).

43. Das, S. et al. ATP citrate lyase improves mitochondrial function in skeletal muscle. Cell Metab. 21, 868-876 (2015).

44. Wallis, R. S. \& Hafner, R. Advancing host-directed therapy for tuberculosis. Nat. Rev. Immunol. 15, 255-263 (2015).

45. Peng, M. et al. Aerobic glycolysis promotes $T$ helper 1 cell differentiation through an epigenetic mechanism. Science 354, 481-484 (2016).

46. Ernst, J. D. The immunological life cycle of tuberculosis. Nat. Rev. Immunol. 12, 581-591 (2012)

47. O'Garra, A. et al. The immune response in tuberculosis. Annu. Rev. Immunol. 31, 475-527 (2013).

48. Chai, Q., Wang, L., Liu, C. H. \& Ge, B. New insights into the evasion of host innate immunity by Mycobacterium tuberculosis. Cell Mol. Immunol 17, 901-913 (2020).

49. Saunders, B. M., Frank, A. A., Orme, I. M. \& Cooper, A. M. Interleukin-6 induces early gamma interferon production in the infected lung but is not required for generation of specific immunity to Mycobacterium tuberculosis infection. Infect. Immun. 68, 3322-3326 (2000). 
50. Chandran, A. et al. Mycobacterium tuberculosis infection induces HDAC1mediated suppression of IL-12B gene expression in macrophages. Front. Cell Infect. Microbiol. 5, 90 (2015).

51. Wang, Z. et al. Combinatorial patterns of histone acetylations and methylations in the human genome. Nat. Genet. 40, 897-903 (2008).

52. Nandy, A. et al. Adipocyte Model of Mycobacterium tuberculosis infection reveals differential availability of iron to bacilli in the lipid-rich caseous environment. Infect. Immun. 86, e00041-18 (2018).

53. Bossche, J. V., O'Neill, L. A. \& Menon, D. Macrophage immunometabolism: where are we (going)? Trends Immunol. 38, 395-406 (2017).

54. Ehrt, S., Schnappinger, D. \& Rhee, K. Y. Metabolic principles of persistence and pathogenicity in Mycobacterium tuberculosis. Nat. Rev. Microbiol. 16, 496-507 (2018).

55. Russell, D. G., Huang, L. \& VanderVen, B. C. Immunometabolism at the interface between macrophages and pathogens. Nat. Rev. Immunol. 19, 291-304 (2019).

56. Nazarova, E. V. et al. Rv3723/LucA coordinates fatty acid and cholesterol uptake in Mycobacterium tuberculosis. Elife 6, e26969 (2017)

57. Wilburn, K. M., Fieweger, R. A. \& VanderVen, B. C. Cholesterol and fatty acids grease the wheels of Mycobacterium tuberculosis pathogenesis. Pathog. Dis. 76 fty021 (2018).
58. Wang, Y. et al. Long noncoding RNA derived from CD244 signaling epigenetically controls CD8+ T-cell immune responses in tuberculosis infection. Proc Natl Acad. Sci. USA 112, E3883-E3892 (2015).

59. Lyu, L. D., Tang, B. K., Fan, X. Y., Ma, H. \& Zhao, G. P. Mycobacterial MazG safeguards genetic stability via housecleaning of 5-OH-dCTP. PLoS Pathog. 9, e1003814 (2013).

60. Thompson, S. et al. Mechanistic studies on beta-ketoacyl thiolase from Zoogloea ramigera: identification of the active-site nucleophile as Cys89, its mutation to Ser89, and kinetic and thermodynamic characterization of wildtype and mutant enzymes. Biochemistry 28, 5735-5742 (1989).

61. Middleton, B. The kinetic mechanism and properties of the cytoplasmic acetoacetyl-coenzyme A thiolase from rat liver. Biochem. J. 139, 109-121 (1974).

62. Zheng, R. et al. Notch4 negatively regulates the inflammatory response to Mycobacterium tuberculosis infection by inhibiting TAK1 activation. J. Infect. Dis. 218, 312-323 (2018).

63. Hu, Y. W. et al. NOD1 deficiency impairs CD44a/Lck as well as PI3K/Akt pathway. Sci. Rep. 7, 2979 (2017).

64. Swaim, L. E. et al. Mycobacterium marinum infection of adult zebrafish causes caseating granulomatous tuberculosis and is moderated by adaptive immunity. Infect. Immun. 74, 6108-6117 (2006).

65. Cronan, M. R. et al. Macrophage epithelial reprogramming underlies mycobacterial granuloma formation and promotes infection. Immunity 45, 861-876 (2016). 